\title{
On the origin and composition of Galactic cosmic rays
}

\author{
N. Prantzos ${ }^{1}$ \\ Institut d'Astrophysique de Paris, UMR7095 CNRS, Univ. P. \& M. Curie, 98bis Bd. Arago, 75104 Paris, France \\ e-mail: prantzos@iap.fr
}

Received 9 June 2011 / Accepted 27 December 2011

\begin{abstract}
Context. The composition of Galactic cosmic rays (GCR) presents strong similarities to the standard (cosmic) composition, but also noticeable differences, the most important of which is the high isotopic ratio of ${ }^{22} \mathrm{Ne} /{ }^{20} \mathrm{Ne}$, which is $\sim 5$ times higher in GCR than in the Sun. This ratio provides key information on the GCR origin.

Aims. We investigate the idea that GCR are accelerated by the forward shocks of supernova explosions, while they run through the pre-supernova winds of the massive stars and through the interstellar medium.

Methods. We use detailed wind and core yields of rotating and non-rotating models of massive stars with mass loss, as well as simple models for the properties of the forward shock and of the circumstellar medium.

Results. We find that the observed $\mathrm{GCR}^{22} \mathrm{Ne} /{ }^{20} \mathrm{Ne}$ ratio can be explained if GCR are accelerated only during the early Sedov phase, for shock velocities $>1600 \mathrm{~km} \mathrm{~s}^{-1}$. The acceleration efficiency is found to be of the order of $10^{-6}-10^{-5}$, i.e. a few particles out of a million encountered by the shock escape the SN at GCR energies. We also show quantitatively that the widely publicized idea that GCR are accelerated in superbubbles fails to account for the high ${ }^{22} \mathrm{Ne} /{ }^{20} \mathrm{Ne}$ ratio in GCR.
\end{abstract}

Key words. acceleration of particles - shock waves - ISM: supernova remnants - stars: mass-loss

\section{Introduction}

Supernova (SN) shocks are generally thought to be the main accelerator of the bulk of Galactic cosmic rays (GCR). Indeed, the power of GCR in the Milky Way is estimated to be $10^{41} \mathrm{erg} / \mathrm{s}$, corresponding to $10-20 \%$ of the kinetic power of Galactic supernovae (assuming canonical values of $2 \mathrm{SN}$ per century, each one releasing $10^{51} \mathrm{erg}$ of kinetic energy).

The site of the acceleration of GCR remains debatable today, despite more than five decades of theoretical and observational studies (e.g. Strong et al. 2007, and references therein). Over the years, it has been suggested that GCR are accelerated in 1) SN remnants (either by the forward or the reverse shock or both), 2) the interstellar medium (ISM), 3) the winds of massive stars, 4) the interiors of superbubbles, excavated by the massive star winds and the subsequent $\mathrm{SN}$ explosions of an $\mathrm{OB}$ association.

Each one of the proposed sites has its own advantages and shortcomings, regarding the energetics and/or the composition of accelerated matter. For instance, it has been argued that the hot, low-density environment of a superbubble minmizes radiative losses of SN shocks and energy losses of accelerated particles, thus allowing the latter to reach substantial energies, up to the "knee" of the GCR spectrum (e.g. Parizot et al. 2004). On the other hand, reverse SN shocks running into the SN interior carry insufficient energy to explain the bulk GCR energetics (Ramaty et al. 1997). Moreover, they should accelerate ${ }^{59} \mathrm{Ni}$, a product of explosive nucleosynthesis that is unstable to $\mathrm{e}^{-}$-capture (with a lifetime of $10^{4} \mathrm{yr}$ ) and that has not been detected in GCR (Wiedenbeck et al. 1999), while a rapid acceleration would render it practically stable and thus detectable.

It was realized early on that the elemental composition of GCR differs significantly from the one of the ISM. Those differences may provide valuable information on the origin of GCR particles and, perhaps, on the site - and even the mechanism - of acceleration. Volatiles behave differently from refractories: the former display a mass-dependent enrichment with respect to $\mathrm{H}$, which reaches a factor of 10 for the heaviest of them; the latter are all overabundant (w.r.t. H) by a factor of 20 , while $\mathrm{C}$ and O display intermediate overabundances, by factors of 9 and 5, respectively (e.g. Wiedenbeck 2007, and references therein).

This complex pattern is now thought to result not from ionization effects (as suggested in Cassé \& Goret 1978, and further developed by Meyer 1985) but rather from effects related to elemental condensation temperature (Meyer et al. 1997): refractories are locked in dust grains, which are sputtered by repeated SN shocks and the released ions are easily picked-up and accelerated (Ellison et al. 1997). This quite elaborate scheme, which builds on earlier ideas by e.g. Cesarsky \& Bibring (1981), accounts quantitatively for most of the observed features of GCR source composition; still, it leaves unanswered the key question about the acceleration site of GCR (and how it affects the composition of accelerated matter).

The most conspicuous feature of GCR source composition is undoubtely the high isotopic ${ }^{22} \mathrm{Ne} /{ }^{20} \mathrm{Ne}$ ratio. Its value was measured since the late 1970ies (Garcia-Munoz et al. 1979; Wiedenbeck \& Greiner 1981). The most accurate measurement today, obtained from analysis of the CRIS instrument, leads to a best estimate (Binns et al. 2008) of $0.387 \pm 0.007$ (statistical) \pm 0.022 (systematic). This is $5.3 \pm 0.3$ times the value of the $\left({ }^{22} \mathrm{Ne} /{ }^{20} \mathrm{Ne}\right)_{\odot}$ ratio in the solar wind. In contrast to the case of the elemental source GCR abundances, which may be affected by various physico-chemical factors (first ionization potential, condensation temperature, etc.) isotopic ratios can only be affected by nucleosynthetic processes and hence provide crucial information on the origin of cosmic ray particles. It should be noticed that up to now there is no clear evidence for any other GCR isotopic ratio to differ from solar, with the potential exception of 
${ }^{58} \mathrm{Fe} /{ }^{56} \mathrm{Fe}$, which is estimated to be $1.687 \pm 0.274$ times solar (Binns et al. 2008).

Soon after the discovery of the anomalous GCR ${ }^{22} \mathrm{Ne} /{ }^{20} \mathrm{Ne}$ ratio, Cassé \& Paul (1982) suggested that it could be explained by a mixture of $\sim 2 \%$ of material from the wind of a WC star to $98 \%$ of material with standard composition. In early Heburning, ${ }^{14} \mathrm{~N}$ (produced through the CNO cycle in the previous $\mathrm{H}$-burning phase) is transformed almost totally in ${ }^{22} \mathrm{Ne}$ through ${ }^{14} \mathrm{~N}(\alpha, \gamma){ }^{18} \mathrm{~F}\left(\beta^{+}\right){ }^{18} \mathrm{O}(\alpha, \gamma){ }^{22} \mathrm{Ne}$. He-burning products (like ${ }^{12} \mathrm{C}$ and ${ }^{22} \mathrm{Ne}$ ) are expelled by the stellar winds of massive stars during their WC phase. The observed GCR ${ }^{22} \mathrm{Ne} /{ }^{20} \mathrm{Ne}$ ratio is obtained by assuming dilution of WC material with matter of standard composition. Subsequent studies put the aforementionned idea on a quantitative basis, with the use of detailed models of the evolution and nucleosynthesis of massive, mass-losing stars (Maeder 1983; Prantzos 1984; Meyer 1985; Prantzos et al. 1987). In those studies, the acceleration site of GCR was considered as decoupled from the nucleosynthesis site, and unrelated to the fraction of admixtured WC material.

Higdon \& Lingenfelter (2003) evaluated quantitatively the ${ }^{22} \mathrm{Ne} /{ }^{20} \mathrm{Ne}$ ratio within a superbubble, created by the collective action of stellar winds and SN shockwaves. They adopted stellar wind yields for ${ }^{20} \mathrm{Ne}$ and ${ }^{22} \mathrm{Ne}$ from the models of Schaller et al. (1992) and SN yields from the models of Woosley \& Weaver (1995). Higdon \& Lingenfelter (2003) found that the ${ }^{22} \mathrm{Ne} /{ }^{20} \mathrm{Ne}$ ratio in the superbubble decreases with time (because ${ }^{22} \mathrm{Ne}$ from the winds dominates the evolution of ${ }^{22} \mathrm{Ne} /{ }^{20} \mathrm{Ne}$ at early times) and that its time average value is compatible with the GCR source ${ }^{22} \mathrm{Ne} /{ }^{20} \mathrm{Ne}$ inferred from observations. In a subsequent paper, Lingenfelter \& Higdon (2007) recognised that the Schaller et al. (1992) yields of ${ }^{22} \mathrm{Ne}$ were highly overestimated ${ }^{1}$ and, consequently, "new detailed calculations of the expected GCR isotopic ratio are called for", but they did not attempt such a re-evaluation. In the meantime, Binns et al. (2005), using updated wind yields of massive stars with rotation (from the Geneva group, see Sect. 2.2), found good agreement between the observed ${ }^{22} \mathrm{Ne} /{ }^{20} \mathrm{Ne}$ ratio and an admixture of $\sim 20 \%$ material from WR stars with $80 \%$ material of standard composition. According to Binns et al. (2008), since WR stars are evolutionary products of OB stars, such an agreement "suggests that OB associations within superbubbles are the likely source of at least a substantial fraction of GCR".

Howewer, theoretical studies in the past 10 years are based mostly on the paradigm of GCR being accelerated in SN remnants, not in superbubbles, e.g. Ptuskin \& Zirakashivili (2005); Berezhko \& Völk (2006); Berezhko et al. (2009); Ptuskin et al. (2010); Caprioli et al. (2010); Schure et al. (2010), Ellison \& Bykov (2011) and references therein. The kinetic energy of the bulk motion of the forward shock of the SN explosion is converted into GCR energy through diffusive shock acceleration. The process is highly non-linear and involves the dynamical reaction of both the accelerated particles and of the magnetic field on the system. Those studies usually take into account that the SN explosion often occurs within the cavity excavated in the interstellar medium (ISM) by the wind of the massive star prior to the explosion, e.g. Biermann et al. (2001); however, the structure of the circumstellar environment in that case is quite complex and simplified models are used for its description. Although Caprioli et al. (2011) considered the composition of GCR $(\mathrm{H}$, $\mathrm{He}, \mathrm{CNO}, \mathrm{MgSiAl}, \mathrm{Fe})$ resulting from such an acceleration site, none of those studies considered the ${ }^{22} \mathrm{Ne} /{ }^{20} \mathrm{Ne}$ ratio.

\footnotetext{
1 The reason was the excessively high mass-loss rates that were adopted in that work.
}

In this work we study the ${ }^{22} \mathrm{Ne} /{ }^{20} \mathrm{Ne}$ ratio of GCR accelerated by the forward shocks of $\mathrm{SN}$ explosions, while they run through the presupernova winds of massive stars and through the interstellar medium. We consider the whole mass spectrum of massive stars (from $\sim 10$ to $120 M_{\odot}$ ), including stars with either small or large mass losses prior to their explosions. We consider stellar properties (masses of winds, ejecta, yields etc.) from recent models with mass loss and or without rotation (from Hirschi et al. 2005; and Limongi \& Chieffi 2006, respectively), the former having greater ${ }^{22} \mathrm{Ne}$ enhancements in their winds. We adopt a simplified prescription (suggested in Ptuskin \& Zirakashvili 2005; and reformulated in Caprioli 2011) to describe the structure of the circumstellar medium at the time of the explosion and we consider that GCR start being accelerated in the Sedov-Taylor (ST) phase of the SN remnant (see e.g. Ptuskin et al. 2010). By requiring the resulting IMF averaged ${ }^{22} \mathrm{Ne} /{ }^{20} \mathrm{Ne}$ ratio to equal the observed one $R_{\mathrm{Obs}}=$ $\left({ }^{22} \mathrm{Ne} /{ }^{20} \mathrm{Ne}\right)_{\mathrm{GCR}} /\left({ }^{22} \mathrm{Ne} /{ }^{20} \mathrm{Ne}\right)_{\odot}=5.3 \pm 0.3$ we are able to constrain the forward shock velocity to values $>1600-1900 \mathrm{~km} \mathrm{~s}^{-1}$ for rotating stars and to $>2100-2400 \mathrm{~km} \mathrm{~s}^{-1}$ for non rotating ones (depending on assumptions on acceleration efficiency), i.e. we find that GCR are accelerated during the early ST phase, lasting for a few $10^{3} \mathrm{yr}$. Assuming that $10 \%$ of the SN kinetic energy is converted to GCR, we find that during the acceleration period a few particles out of a million encountered by the forward shock are accelerated. Finally, we reassess the superbubble paradigm for the origin of GCR, by evaluating consistently the ${ }^{22} \mathrm{Ne} /{ }^{20} \mathrm{Ne}$ ratio with the aforementioned stellar yields. We find that it cannot be as high as observed, unless some extremely favorable assumptions are made (only the early period of the superbubble lifetime considered, no gas left over from the formation of the OB association). We conclude that the bulk of GCR cannot originate in superbubbles.

The plan of the paper is as follows. In Sect. 2 we present the general set-up of our model: the adopted stellar models (Sect. 2.2) and their wind yields (Sect. 2.3), the description of the circumstellar environment (Sect. 2.4) and the evolution of the forward shock in the ST phase (Sect. 2.5). In Sect. 3 we present our results for the (time-dependent) composition of the accelerated particles, the limits imposed on the shock velocity by the observed ${ }^{22} \mathrm{Ne} /{ }^{20} \mathrm{Ne}$ ratio and the efficiency of the particle acceleration. Finally, in Sect. 4 we explore the ${ }^{22} \mathrm{Ne} /{ }^{20} \mathrm{Ne}$ ratio of GCR, assumed to be accelerated inside a superbubble, and we show that it cannot match the oberved one (unless extreme assumptions are made). The results are summarized in Sect. 5.

\section{A toy model for the composition of CR accelerated in massive star winds}

\subsection{The set-up}

The method adopted here to calculate the composition of matter accelerated by a single SN explosion is schematically illustrated in Fig. 1. At the end of its life and at the time of its SN explosion, a star of initial mass $M_{*}$ is left with a mass $M_{\text {Exp }}$, surrounded by a circumstellar shell of mass $M_{\text {Wind }}=M_{*}-M_{\text {Exp }}$, which has been lost through stellar wind during its prior hydrostatic evolution After the SN explosion, a mass of ejecta $M_{\mathrm{Ej}}=$ $M_{\text {Exp }}-M_{\text {Rem }}$ (where $M_{\text {Rem }}$ is the mass of the compact remmant, neutron star or black hole) expands first within the shell of mass $M_{\text {Wind }}$ and then in the ISM, with the forward shock having initial velocity $v_{0}=\sqrt{2 E_{0} / M_{\mathrm{Ej}}}$, where $E_{0}$ is the kinetic energy of the $\mathrm{SN}$ explosion. 


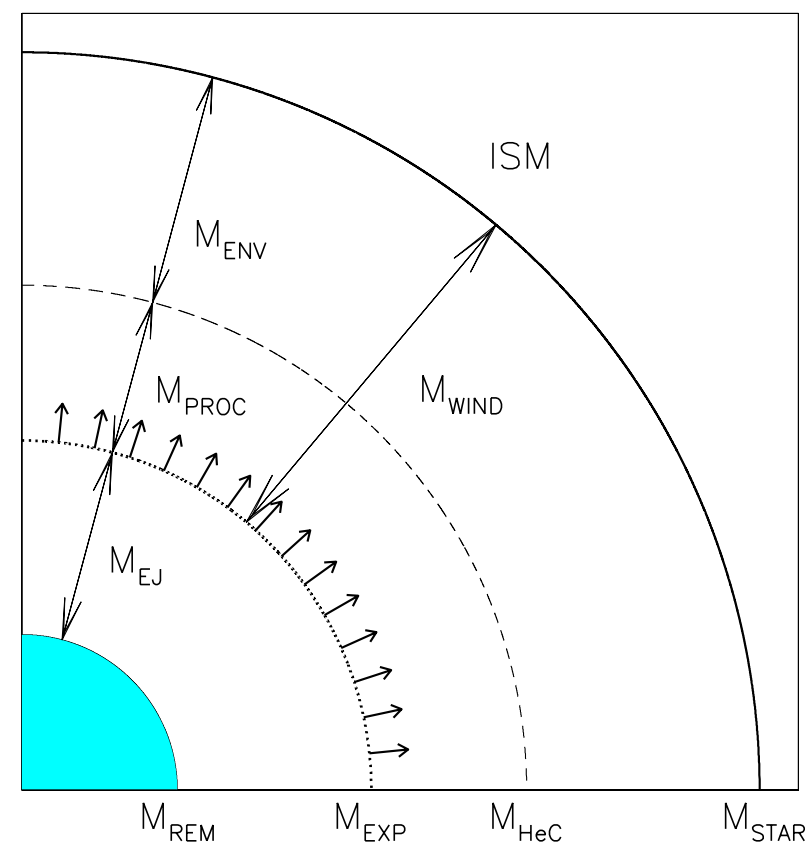

Fig. 1. Schematic representation of a supernova exploding in the wind of its parent star. The star of initial mass $M_{*}$ explodes with a mass $M_{\text {Exp }}$, i.e. it has lost a mass $M_{\text {wind }}=M_{*}-M_{\text {Exp }}$. The most massive stars become WR stars and their wind expels not only the H-envelope (of mass $M_{\mathrm{Env}}$, with composition similar to that of the ISM) but also nuclearly processed layers, of mass $M_{\text {Proc }}$, i.e. $M_{\text {Wind }}=M_{\text {Env }}+M_{\text {Proc }}$, where $M_{\mathrm{Proc}}=M_{\mathrm{HeC}}-M_{\mathrm{Exp}}$ and $M_{\mathrm{HeC}}$ is the mass of the (H-exhausted) He-core. The star leaves a remnant (neutron star or black hole) of mass $M_{\text {Rem }}$; the mass ejected in the SN explosion is $M_{\mathrm{Ej}}=M_{\mathrm{Exp}}-M_{\mathrm{Rem}}$. Efficient GCR acceleration presumably starts at the beginning of the ST phase, when a mass $M_{S 1} \sim M_{\mathrm{Ej}}$ is swept up in front of the SN shock wave (which is indicated by arrows).

For stars ending their lives as WR stars, the wind contains both the original (nuclearly unprocessed) envelope of mass $M_{\text {Env }}$, and nuclearly processed layers of mass $M_{\text {Proc }}$, enriched in products of H-burning, and in some cases of He-burning as well. For those stars, $M_{\text {Wind }}=M_{\text {Proc }}+M_{\text {Env }}$ and $M_{\text {Proc }}$ is calculated as the difference between the mass of the nuclearly processed core $M_{\mathrm{HeC}}$ and the mass at the explosion: $M_{\mathrm{Proc}}=M_{\mathrm{HeC}}-M_{\mathrm{Exp}}$. For lower mass stars that explode as red supergiants, the wind composition results essentially from the 1 st dredge-up, i.e. it is a mixture of $\mathrm{H}$-burning products from the stellar core with the original envelope composition (i.e. mass loss has not uncovered the He-core at the time of the explosion). The limit between the two classes of stars depends on their initial mass, mass-loss rate and rotational velocity and it is rather poorly known at present: in general, in models with no rotation stars with $M_{*}>32-35 M_{\odot}$ become WR stars (e.g. Heger et al. 2002), while in models with rotation that limit may be as low as $22 M_{\odot}$ (Meynet \& Maeder 2000).

The first phase of the supernova remnant ("free expansion") takes place at shock velocity $v \sim$ const. and ends when a mass $M_{\mathrm{S} 1} \sim M_{\mathrm{Ej}}$ has been swept up in front of the shock wave, at which point the ST phase sets in. Following Ptuskin et al. (2010), we assume that efficient GCR acceleration starts at this time, where the situation is energetically most favourable. In our baseline model we consider constant acceleration efficiency ; timedependent efficiency of particle acceleration is the subject of current researches (see Ellison \& Bykov 2011; Drury 2011, and references therein) and will be briefly discussed in Sect. 3.3.
The ST phase proceeds adiabatically, i.e. at about constant energy and with decreasing velocity, until the temperature of the gas engulfed by the shock front drops to levels allowing a significant fraction (about 50\%) of the remaining energy to be radiated away. At that time, an amount of matter $M_{\mathrm{S} 2} \gg M_{\mathrm{Ej}}$ has been swept up and the shock enters the "snow-plow" phase. At this point - and, perhaps, even earlier, during the ST phase - the forward shock is too weak to accelerate particles to GCR energies any more.

In the aforementioned scenario, GCR are accelerated from a pool of particles with composition characteristic of the mass $M_{\text {Wind }}$ early on. Depending on the initial stellar mass, this composition may be rich in products of $\mathrm{H}$ - (and He-) burning. It is progressively diluted with ambient (first wind - with normal ${ }^{22} \mathrm{Ne}-$ and then interstellar) gas and at the end of the ST phase it closely resembles closely that of the ISM. The GCR source composition observed on Earth should correspond to the average composition between the early ST phase and some later evolutionary stage of the remnant, and should result from the whole mass spectrum of exploding stars, i.e. it should be averaged over a stellar initial mass function (IMF).

\subsection{Properties of mass-losing stars}

We adopt two sets of stellar models in this work. They are calculated for stars of solar metallicity, and in both cases the solar mixture of Anders \& Grevesse (1979) is adopted. The corresponding metallicity is $Z_{\odot}=0.019$, substantially higher than more recent values (Lodders 2003; Asplund et al. 2010) and this difference results in particular from the reduction in the past decade of the solar abundances of $\mathrm{C}, \mathrm{N}, \mathrm{O}$ and $\mathrm{Ne}$, which are key elements for the purpose of this work. For obvious consistency reasons, we keep here the Anders \& Grevesse (1979) values, when comparing our results for GCR to solar ones.

The first set of stellar models is the one of the Frascati group (Limongi \& Chieffi 2006, hereafter LC06). It comprises 15 model stars between 11 and $120 M_{\odot}$ with mass loss but no rotation. The model includes all stages of hydrostatic nuclear burning and simulates the final stellar explosion by imparting an initial velocity to a mass coordinate of $1 M_{\odot}$ (i.e. well inside the $\mathrm{Fe}$ core of the stars); the mass cut (the limit separating the ejecta of mass $M_{\mathrm{Ej}}$ from the compact remnant of mass $M_{\mathrm{Rem}}$ ) is chosen such that $0.1 M_{\odot}$ of ${ }^{56} \mathrm{Ni}$ is ejected by the explosion.

The various masses involved in the "toy model" of Sect. 2.1 and Fig. 1 are provided in Table 2 of LC06 and are displayed in Fig. 2 (top left) of this work, whereas derived quantities are displayed in Fig. 2 (bottom left). It can be seen that stars with $M_{*}<20 M_{\odot}$ have lost a negligible amount of mass prior to the explosion $\left(M_{\mathrm{Wind}}<M_{\mathrm{Ej}}\right)$ and the ST phase starts within the ambient ISM. Stars with $M_{*}>30 M_{\odot}$ have $M_{\mathrm{HeC}}>M_{\text {Exp }}$, i.e. they explode as WR stars, having expelled nuclearly processed layers in their winds. But only for the most massive stars $\left(M_{*}>60 M_{\odot}\right)$ one has $M_{\mathrm{Ej}}<M_{\text {Proc }}$, i.e. in the beginning of the ST phase the shock wave still expands into material with a composition reflecting that of the nuclearly processed core; for lower mass stars $\left(30<M_{*} / M_{\odot}<60\right)$ the ST phase starts when the shock wave encounters material with mixed composition from the core and the envelope, i.e. less enriched in $\mathrm{H}$ - and $\mathrm{He}$ - burning products. An interesting feature of those models is that the mass of the ejecta $M_{\mathrm{Ej}} \sim 10 M_{\odot}$ is similar for all of them, leading to similar properties (duration, swept-up mass) for their corresponding ST phases. 
A\&A 538, A80 (2012)

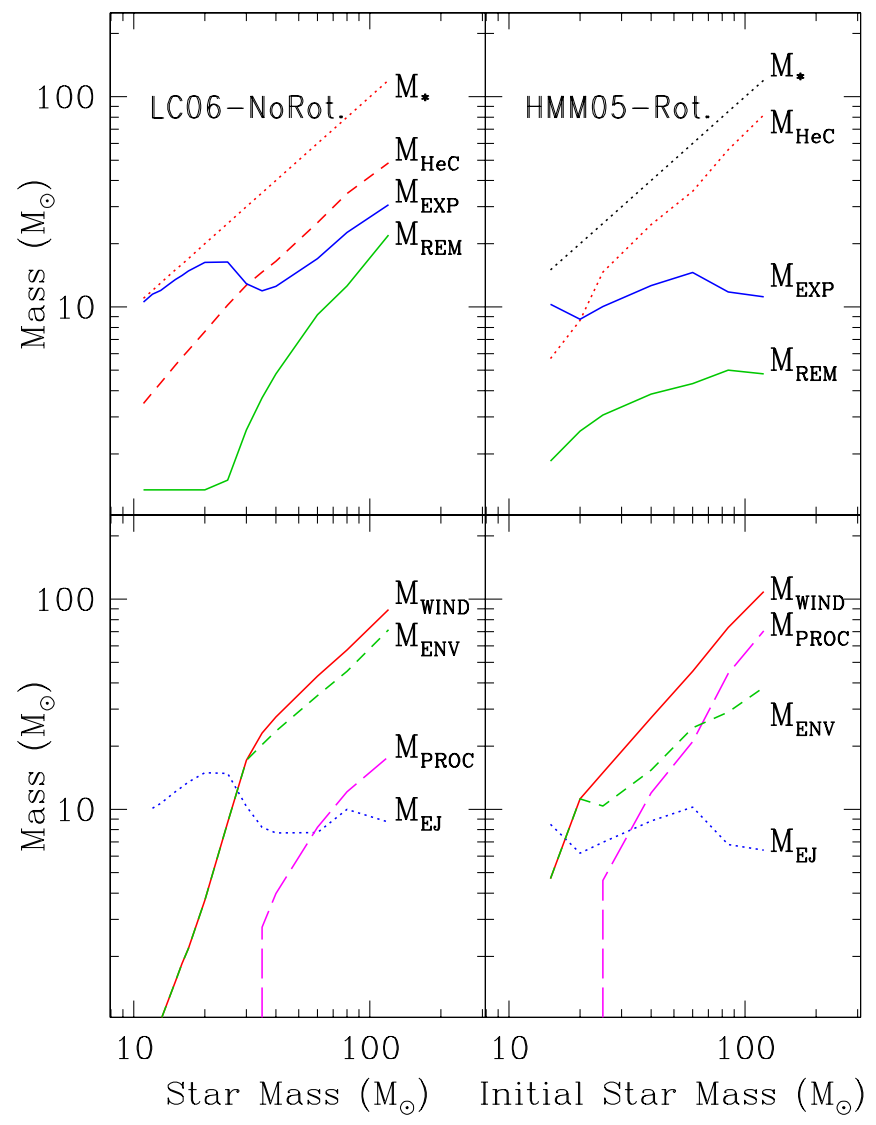

Fig. 2. Input data adopted in this work for solar metallicity massive stars with mass loss. Left: non rotating models from Limongi \& Chieffi (2006); right: rotating models from Hirschi, Meynet \& Maeder (2005). Top: mass of the (H-exhausted) $\mathrm{He}$ core $M_{\mathrm{HeC}}$, of the star at explosion $M_{\text {Exp }}$ and of the compact remnant $M_{\text {Rem }}$ as a function of the initial stellar mass $M_{*}$. Bottom: derived quantities are the mass of the wind $M_{\text {Wind }}=M_{*}-M_{\text {Exp }}$, the mass of the nuclearly processed layer $M_{\text {Proc }}=M_{\mathrm{HeC}}-M_{\mathrm{Rem}}$ and of the unprocessed ${ }^{22} \mathrm{Ne}$-normal) envelope $M_{\text {Env }}=M_{\text {Wind }}-M_{\text {Proc }}$ and the mass of the SN ejecta $M_{\text {Ej }}=M_{\text {Exp }}-M_{\text {Rem }}$ In all cases, curves are smoothly interpolated between model results.

The second set of stellar models is that of the Geneva group, calculated by Hirschi et al. (2005, hereafter HMM05) and includes both mass loss and rotation for stars with mass between 12 and $60 M_{\odot}$; it has been complemented with results for stars of 85 and $120 M_{\odot}$, kindly provided by Meynet (priv. comm.). The initial rotational velocity is $v_{\text {Rot }}=300 \mathrm{~km} \mathrm{~s}^{-1}$ on the ZAMS, corrrsponding to an average velocity of $220 \mathrm{~km} \mathrm{~s}^{-1}$ on the main sequence, i.e. close to the average observed value. Non-rotating models with the same physical ingredients (for convection, mass loss, etc.) have also been calculated for comparison. The evolution has been calculated to the end of Si-burning but the final $\mathrm{SN}$ explosion was not considered. Instead, an empirical prescription was used to evaluate the mass of the compact remnant. The masses of our "toy model", as given in Table 2 of HMM05, are displayed in Fig. 2. There is an important difference with respect to Table 2 of HMM05: they provide the mass of He-core at the time of the explosion and for the most massive stars this coincides with $M_{\operatorname{Exp}}$ (i.e. the mass left to the star at explosion is smaller than the maximum extent of the He-core); however, we are interested in the true value of $M_{\mathrm{HeC}}$ (since this will determine how much mass of processed material the shock wave will encounter) and that value is obtained through the detailed results of
HMM05 (displaying the wind composition as a function of time - or of mass left).

Comparing the results of LC06 and HMM05 one sees that rotation increases the mass loss $\left(M_{\text {Wind }}\right.$ larger in HMM05), thus leaving the star with a smaller mass at explosion $\left(M_{\operatorname{Exp}}\right.$ smaller in HMM05). Rotation also increases the size of nuclearly processed regions $\left(M_{\mathrm{HeC}}\right.$ larger in HMM05), because matter is rotationally mixed outwards to greater distances than achieved through convection. In turn, this leads to larger amounts of processed material $M_{\text {Proc }}$ for the HMM05 models.

The aforementioned features of rotating vs non-rotating models, which are explained in detail in e.g. Maeder \& Meynet (2000) are crucial for understanding the differences in the corresponding wind yields of the stars.

\subsection{The wind composition of massive stars}

LC06 provided (private communication) yields $y_{i}$ of all stable nuclear species, from $\mathrm{H}$ to $\mathrm{Ge}$, included in their models and ejected through the winds of the stars, up to the moment of the explosion. HMM05 provide (Table 3 in their paper) the net yields $y_{n, i}$ of the winds of their models for stable species from ${ }^{3} \mathrm{He}$ to ${ }^{23} \mathrm{Na}$, from which the yields can be recovered through

$y_{i}\left(M_{*}\right)=y_{n, i}\left(M_{*}\right)+M_{\text {Wind }} X_{\odot, i}$,

where the adopted solar values $X_{\odot, i}$ are displayed in Table 1 of HMM05.

The wind yields of a few selected species appear in Fig. 3 for the non-rotating models of LC06 and for both the non-rotating and the rotating models of HMM05. It can be seen that the results for non-rotating models of LC06 and HMM05 generally agree very well for stars up to $40 M_{\odot}$. Their results differ only for the $60 M_{\odot}$ model (and presumably for higher masses as well) and only for the He-burning products ${ }^{12} \mathrm{C},{ }^{16} \mathrm{O}$ and ${ }^{22} \mathrm{Ne}$. Because both HMM05 and LC06 use the same prescriptions for mass loss, the reason for that discrepancy could be the use of a small amount of overshooting in HMM05.

Rotation has a twofold effect on stellar yields: it increases the size of the nuclearly processed layers (because it mixes material to greater distances than convection alone) and reduces the escape velocity in the stellar equator, allowing larger amounts of mass to be ejected in the wind. Both effects enhance the wind yields up to a certain mass limit; above it, the wind has removed so much mass that less material is left in the star to be processed in subsequent stages of the evolution, thus reducing the corresponding yields. This is the case, for instance, with the He-burning products ${ }^{12} \mathrm{C}$ and ${ }^{22} \mathrm{Ne}$, the yields of which decrease above $\sim 60 M_{\odot}$ in the rotating HMM05 models (see Fig. 3 and HMM05 for details).

In the following we assume that the wind interaction with the ISM has not substantially changed the wind stratification: the forward shock will first encounter the innermost wind layers, which contain processed material in the most massive stars; later it will encounter the outer wind layers (containing mostly the initial composition), before running into the ISM. Figure 4 displays the mass integrated composition of the wind (for a few key metals), as encountered by the forward shock, moving outwards from $M_{\text {Exp }}$, for two rotating model stars of $25 M_{\odot}$ and $60 M_{\odot}$ (from HMM05). The quantity

$m_{i}(M)=\int_{M_{\text {Exp }}}^{M_{*}} X_{\mathrm{Wind}, i}(M) \mathrm{d} M$,

is displayed as a function of mass coordinate $M, X_{\text {wind }, i}(M)$ being the mass fraction of isotope $i$ in the wind of the star of mass $M_{*}$. 


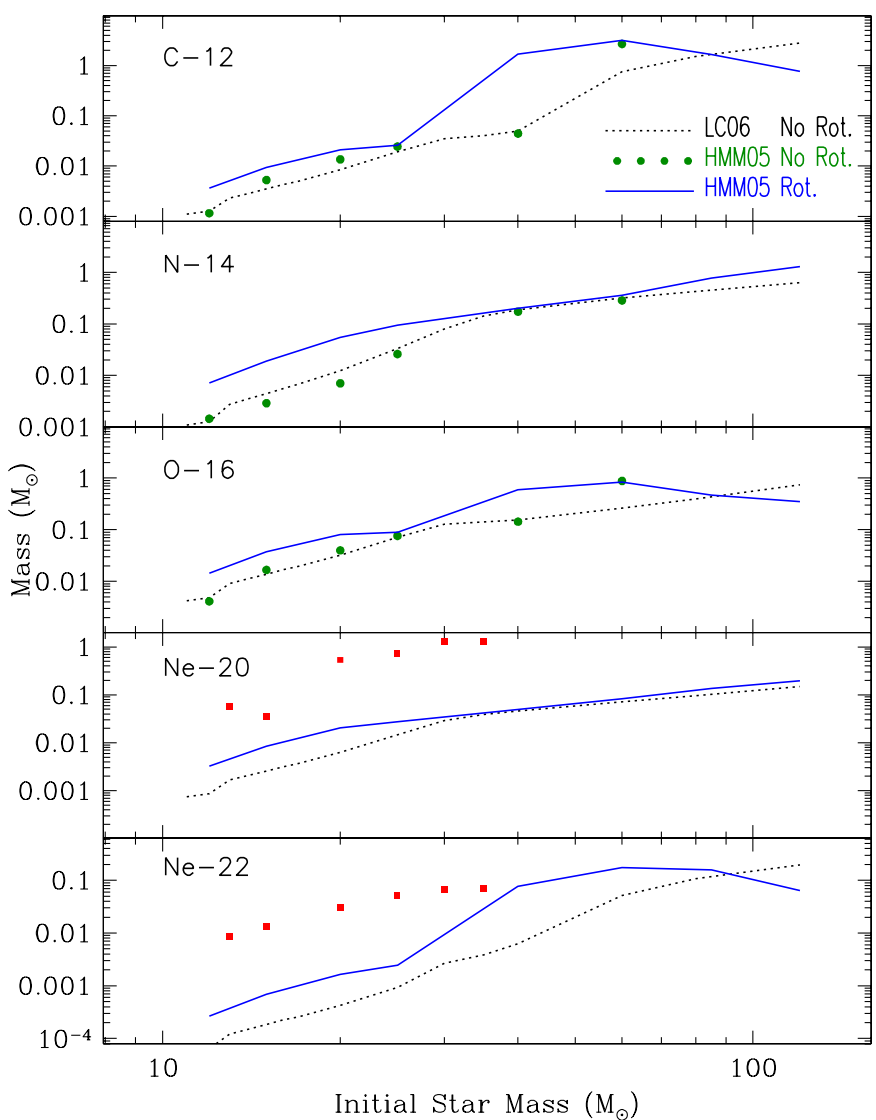

Fig. 3. Total masses of selected isotopes in the winds of the massive stars from the two sets of models adopted in this work. Solid curves are yields from HMM05 (with rotation) and dotted curves from LC06 (no rotation), in both cases interpolated between model results. The points correspond to results of the HMM05 models with no rotation and, in general, agree well with LC06 results except for the $60 M_{\odot}$ star.

Obviously, one has $m_{i}\left(M_{\operatorname{Exp}}\right)=0$. and $m_{i}\left(M_{*}\right)=y_{i}\left(M_{*}\right)$, i.e. at crossing the last (outermost) wind layer, the forward shock has encountered the totality of the yield $y_{i}\left(M_{*}\right)$.

An inspection of Fig. 4 shows that

- for the $25 M_{\odot}$ star (no He-burning products encountered by the shock wave), the innermost layers contain most of the ${ }^{14} \mathrm{~N}$ produced by the CNO cycle (its mass increases slowly in the outermost layers), and little of ${ }^{12} \mathrm{C}$ and ${ }^{16} \mathrm{O}$, which are depleted by the $\mathrm{CNO}$ cycle (their mass increases rapidly in the outermost layers); ${ }^{20} \mathrm{Ne}$ and ${ }^{22} \mathrm{Ne}$ are little affected by $\mathrm{H}-$ burning and their integrated wind mass increases in a way intermediate between ${ }^{14} \mathrm{~N}$ and ${ }^{12} \mathrm{C}$.

- for the $60 M_{\odot}$ star (He-burning, then H-burning products encountered by the shock wave), the quasi-totality of the ${ }^{12} \mathrm{C}$ and ${ }^{22} \mathrm{Ne}$ produced by He-burning are encountered in the inner layers (inside $\sim 25 M_{\odot}$ ), while essentially no ${ }^{14} \mathrm{~N}$ is left in that region; the majority of ${ }^{14} \mathrm{~N}$ is found in the region $25<M / M_{\odot}<50$ while substantial ${ }^{16} \mathrm{O}$ is found in the unprocessed envelope (beyond $50 M_{\odot}$ ). Finally, ${ }^{20} \mathrm{Ne}$ is little affected everywhere (almost constant mass fraction) and its integrated mass rises in step with the wind mass of the star.

If detailed information (i.e. wind composition as function of wind mass coordinate) is available, the composition of the material swept-up by the forward shock can be calculated in a consistent way. If only the wind yields $y_{i}$ and the various characteristic

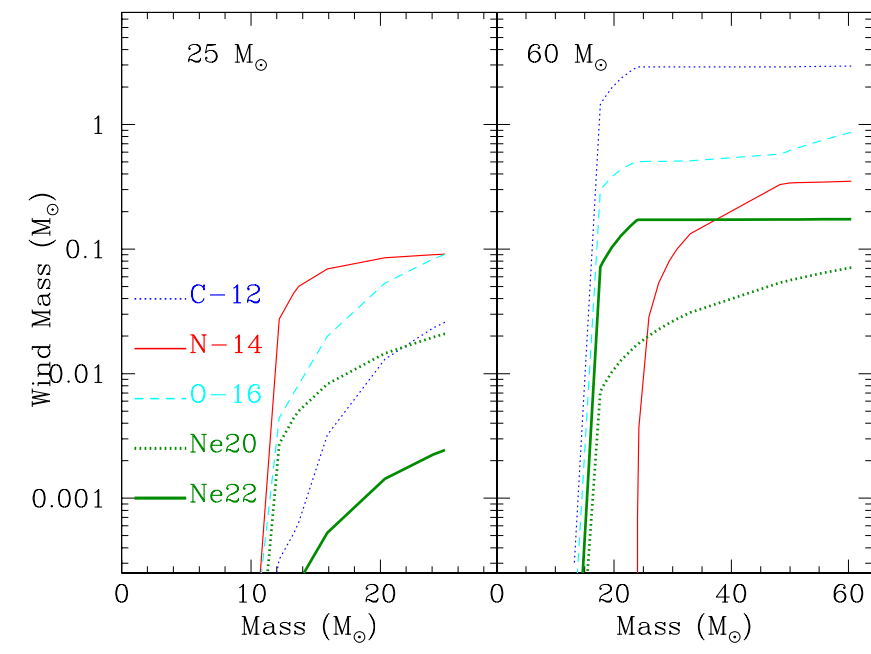

Fig. 4. Composition encountered by the forward shock wave as it moves (from left to right) through the winds of $25 M_{\odot}$ (left) and $60 M_{\odot}(r i g h t)$ rotating stars. The shock wave starts at $M_{\text {Exp }}=10 M_{\odot}$ and $14.8 M_{\odot}$, respectively, and the ST phase sets in when a mass $M_{S 1}=M_{\text {Exp }}$ has been swept up and has presumably been well mixed. The quantities displayed correspond to the mass integrated composition of the wind, starting at $M_{\operatorname{Exp}}$.

masses of Fig. 2 are available, one may adopt the following approximations:

(i) stars that never reach the WR stage $\left(M_{*}<22 M_{\odot}\right.$ for rotating models and $M_{*}<34 M_{\odot}$ for non rotating ones) have their envelopes fully mixed after the 1st dredge-up episode in the red supergiant phase; the wind composition is then simply obtained as

$X_{\text {wind }, i}\left(M_{*}\right)=\frac{y_{i}\left(M_{*}\right)}{M_{\text {Wind }}\left(M_{*}\right)}$,

(ii) stars that do not reach the WR stage first eject their unprocessed envelope of mass $M_{\mathrm{Env}}$ and composition $X_{\mathrm{env}, i}=$ $X_{\mathrm{ISM}, i}$ and then the innermost processed layers of mass $M_{\text {Proc }}$ and composition $X_{\mathrm{PROC}, i}$ such that

$y_{i}\left(M_{*}\right)=X_{\mathrm{Env}, i} M_{\mathrm{env}}\left(M_{*}\right)+X_{\mathrm{Proc}, i} M_{\mathrm{Proc}}\left(M_{*}\right)$,

which allows one to derive $X_{\mathrm{Proc}, i}$, i.e. an average abundance in the processed layers, and obtain thus an average wind composition profile

$$
\begin{aligned}
& X_{\text {Wind }, i}(M)=X_{\mathrm{Proc}, i}\left(M_{*}\right) \text { for } M_{\mathrm{Exp}}<M<M_{\mathrm{HeC}} \\
& X_{\text {Wind }, i}(M)=X_{\mathrm{Env}, i}\left(M_{*}\right) \text { for } M_{\mathrm{HeC}}<M<M_{*} .
\end{aligned}
$$

The method outlined here turns out to provide a good approximation for the detailed composition profiles of the HMM05 models and was used here for the two sets of LC06 and HMM05 models for consistency reasons. Taking into account all the uncertainties of the models (prescriptions for mass loss, convective and rotational mixing etc.), we consider the aforementioned approximation to be fairly satisfactory for the purpose of this work.

\subsection{The circumstellar environment of massive stars}

According to the ideas outlined in Sect. 2.1, particle acceleration to GCR energies starts when a mass $M_{\mathrm{S} 1} \sim M_{\mathrm{Ej}}$ has been sweptup by the forward shock. The composition of that material at 
the very beginning of particle acceleration is that of the material located at mass coordinate $A_{1}=M_{\mathrm{Exp}}+M_{\mathrm{Ej}}$ and obviously constitutes an extreme for the abundances of the corresponding elements (upper limit for those produced in the stellar interior and lower limit for those destroyed, like $\mathrm{H}$ ). In the following we shall assume that at the time of the explosion the stellar wind has fully kept the stratification of its various layers, as they were progressively leaving the stellar surface.

The circumstellar region hit by the forward shock has a complex structure that depends on the properties of the exploded star. That structure has been explored in some detail with hydrodynamical models by Garcia-Segura et al. (1996a,b) for nonrotating stars. In the case study of a $35 M_{\odot}$ star, they have found that the rapid wind of the $\mathrm{O}$ star excavates a large bubble (radius $\sim 36 \mathrm{pc}$ ) of low density $\left(10^{-3} \mathrm{~cm}^{-3}\right)$. Inside it propagates a dense $\left(\sim 1 \mathrm{~cm}^{-3}\right)$, slow wind - released during the red supergiant phase - which occupies the innermost few pc (depending on its assumed velocity, of the order of a few tens of $\mathrm{km} \mathrm{s}^{-1}$ ). The subsequent fast $\left(\sim 10^{3} \mathrm{~km} \mathrm{~s}^{-1}\right)$ WR wind compresses the RSG wind, and most of the mass of the latter is found within a thin shell.

The aforementioned study illustrates the complexity of the situation, but its results can hardly be generalized to the whole mass range of massive stars (for instance, lower mass stars will not display the fast WR wind). Indeed, its results cannot even be safely used for the $35 M_{\odot}$ star, since they depend so critically on the adopted parameters of the model (mass loss rates and wind velocities for the various stages). And they certainly fail to describe the situation for rotating stars, which display slow but intense (and not radially symmetric) mass losses on the main sequence.

In view of these uncertainties, we adopt here a simplified prescription for the structure of the circumstellar bubble, assuming spherical symmetry in all cases. We assume that the winds have excavated a bubble of mean density $n_{0}=0.1 \mathrm{~cm}^{-3}$ and, consequently, of radius

$R_{B}=\left(\frac{3 M_{\mathrm{W}}}{4 \pi \rho_{0}}\right)^{1 / 3}$

with $\rho_{0}=n_{0} m_{\mathrm{p}}, m_{\mathrm{p}}$ being the proton mass. Inside the bubble, the density profile is $\rho(r) \propto r^{-2}$, i.e. it corresponds to a steady stellar wind with mass loss rate $\dot{M}_{\mathrm{W}}$ and velocity $v_{\mathrm{W}}$, which is given by

$\rho(r)=\frac{\dot{M}_{\mathrm{W}}}{4 \pi v_{\mathrm{W}} r^{2}}$.

Our choice of $\rho_{0}$ automatically fixes the $\rho(r)$ profile and corresponds to a combination of $\dot{M}_{\mathrm{W}}$ and $v_{\mathrm{W}}$ values. Obviously, one has

$M_{\mathrm{W}}=\int_{0}^{R_{\mathrm{W}}} \rho(r) \mathrm{d} r$.

Outside $R_{\mathrm{W}}$ we assume an ISM with constant density $\rho_{\mathrm{ISM}}=$ $1 m_{\mathrm{p}} \mathrm{cm}^{-3}$. Our approach is similar to Caprioli (2011), but we do not consider here the more complicate case of a WR wind overtaking a RSG wind.

\subsection{Evolution during the Sedov-Taylor (ST) phase}

We follow the propagation of the forward shock first through the wind bubble and then through the ISM with a simple model presented in Ptuskin \& Zirakashvili (2005) and, in a more concise form, in Caprioli (2011, his Eqs. (3.4) to (3.9)). We start the calculation from the "free-expansion" (ejecta-dominated) phase, where the swept-up mass is smaller than $M_{\mathrm{Ej}}$ and which can be described by self-similar analytical solutions. In the subsequent ST phase (swept-up mass $<M_{\mathrm{Ej}}$ ), the model is based on the "thin shell" approximation (e.g. Ostriker \& McKee 1988) which assumes that the swept-up mass is concentrated in a thin shell behind the shock. We solve numerically the time-dependent equations for continuity of mass, energy and momentum, to recover shock radius and velocity as a function of time. Unlike Caprioli (2011), we assume full adiabaticity in the ST phase, i.e. we do not take into account the 10-20\% energy losses of the shock through $\mathrm{CR}$ acceleration, which would reduce the shock velocity $\left(\propto E_{0}^{0.5}\right)$ by less than $10 \%$. The mass swept-up in the ST phase inside shock radius $R_{\mathrm{S}}$ is

$M_{\mathrm{S}}\left(<R_{\mathrm{S}}\right)=M_{\mathrm{EJ}}+4 \pi \int_{R_{\mathrm{S} 1}}^{R_{\mathrm{S}}} \rho(r) r^{2} \mathrm{~d} r$.

We follow the evolution all the way through the ST phase, which ends when a significant fraction of the energy of the cooling remnant is radiated away (through recombination emission); for a solar mixture this occurs at time

$t_{\mathrm{S} 2} \sim 4.4 \times 10^{4} \mathrm{yr}\left(\frac{E_{0}}{10^{51} \mathrm{erg}}\right)^{2 / 9}\left(\frac{n_{\mathrm{ISM}}}{\mathrm{cm}^{3}}\right)^{-5 / 9}$.

In the framework of this simple model we are able to calculate the composition of the material encountered (and presumably accelerated) by the forward shock as a function of time, or of the swept-up mass: indeed, the integrated mass of each element swept up by the forward shock is given by an equation similar to Eq. (2),

$m_{i}\left(M_{\mathrm{S}}\right)=\int_{M_{\exp }}^{M_{\mathrm{S}}\left(<R_{\mathrm{S}}\right)} X_{i}\left(M_{\mathrm{S}}\right) \mathrm{d} M_{\mathrm{S}}$,

where $X_{i}\left(M_{\mathrm{S}}\right)=X_{\text {wind }, i}$ for $M_{\mathrm{S}}<M_{*}\left(\right.$ Sect. 2.3) and $X_{\mathrm{i}}(M)=$ $X_{\mathrm{ISM}, i}$ for $M_{\mathrm{S}}>M_{*}$, i.e. when the shock propagates into the ISM; the upper limit in the integral is given by Eq. (10).

Equation (12) allows one to link the stellar model, i.e. the abundance profiles $X(M)$, to the evolution during the ST phase through Eq. (10), and to the properties of the shock wave. Since the mass $M_{S 2}$ swept-up in the end of the ST phase is much larger than the wind mass in all cases (a few $10^{3} M_{\odot}$, compared to $\sim 100 M_{\odot}$ at most) for the largest part of the ST phase the swept up material has ISM composition. In order to obtain significant deviations from the solar composition, such as the observed ${ }^{22} \mathrm{Ne} /{ }^{20} \mathrm{Ne}$ ratio, one should assume that significant acceleration occurs only in the early ST phase, when the forward shock is stronger and its velocity higher.

\section{Results}

\subsection{Composition of matter in the ST phase}

Figure 5 (top) displays the evolution of the velocity $v_{\mathrm{S}}$ and radius $R_{\mathrm{S}}$ of the forward shock and of the mass $M_{\mathrm{S}}\left(<R_{\mathrm{S}}\right)$ swept up by it, for a 20 and a $60 M_{\odot}$ rotating star, respectively. The density of the unperturbed ISM is taken to be $1 \mathrm{~cm}^{-3}$ in all cases.

The similarity of the curves for $v_{\mathrm{S}}, R_{\mathrm{S}}$ and $M_{\mathrm{S}}\left(<R_{\mathrm{S}}\right)$ for the 20 and $60 M_{\odot}$ stars simply reflects the self-similarity of the ST solution. The small differences in the early ST phase are caused by the difference of the ejected mass $M_{\mathrm{Ej}}$ in the two stars (6 vs. $10 M_{\odot}$, see Fig. 2$)$, since the other parameters $\left(E_{0}\right.$ and $\left.n_{\mathrm{ISM}}\right)$ are the same. 


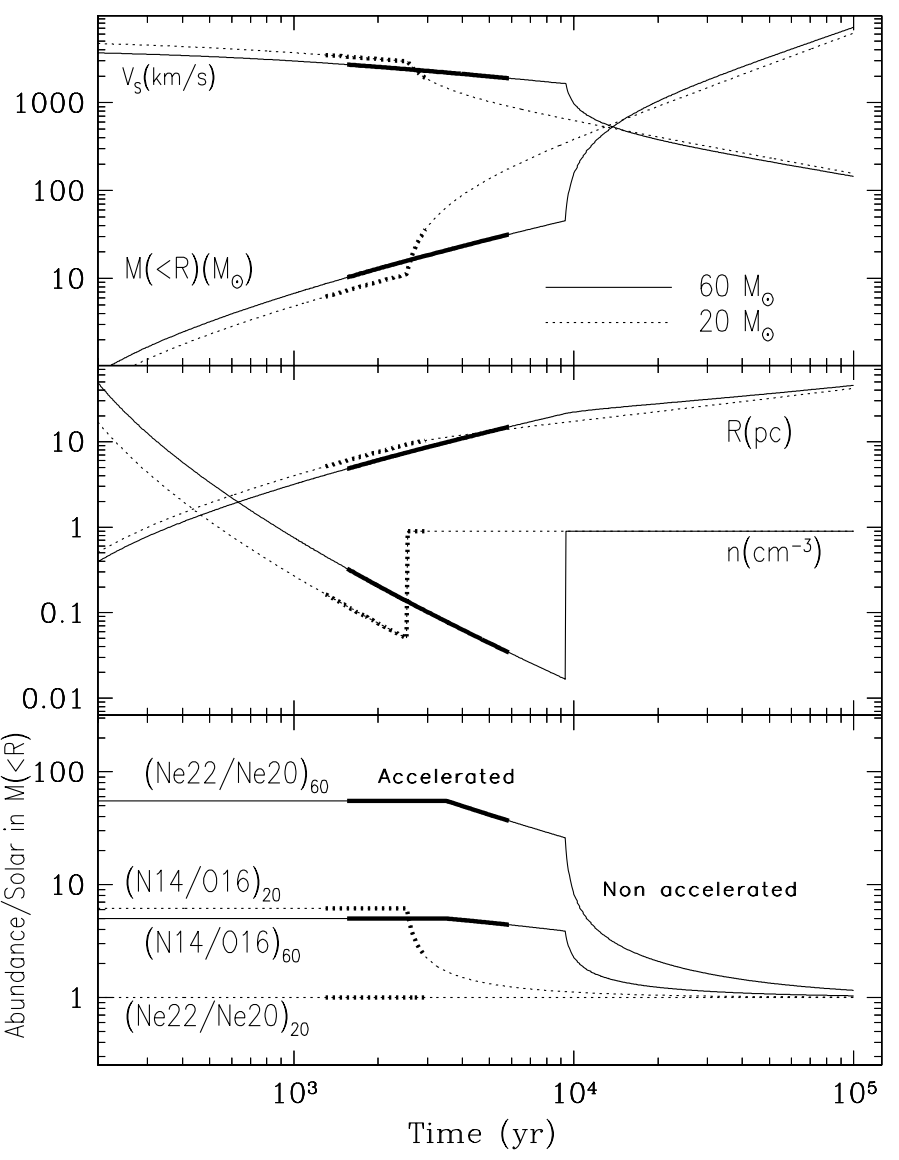

Fig. 5. Evolution of the SN remnants of two rotating stars of initial masses $20 M_{\odot}($ dotted $)$ and $60 M_{\odot}($ solid), respectively. Top: velocity of the forward shock and swept-up mass. Middle: shock radius and density profile before shock arrival (see text). Bottom: composition of the matter swept up up to time $t$ by the shock wave, for the ${ }^{14} \mathrm{~N} /{ }^{16} \mathrm{O}$ and ${ }^{22} \mathrm{Ne} /{ }^{20} \mathrm{Ne}$ ratios. In all panels, the thick portions of the curves indicate the period of efficient particle acceleration, i.e. from the beginning of the ST phase and as far as $v_{\mathrm{s}}>v_{\min }$. The value of $v_{\min }\left(=1900 \mathrm{~km} \mathrm{~s}^{-1}\right.$ for the rotating star models in the figure) is chosen so that the IMF averaged theoretical ratio of ${ }^{22} \mathrm{Ne} /{ }^{20} \mathrm{Ne}$ matches the observed one in GCR (see text, Eq. (13) and Fig. 6).

The bottom panel of Fig. 5 displays the evolution of the ${ }^{14} \mathrm{~N} /{ }^{16} \mathrm{O}$ and ${ }^{22} \mathrm{Ne} /{ }^{20} \mathrm{Ne}$ ratios (i.e. the ratios of the corresponding masses of Eq. (12) for each isotope), expressed in units of the corresponding solar values. In both cases, the forward shock first encounters layers with low ${ }^{16} \mathrm{O}$ and high ${ }^{14} \mathrm{~N}$, resulting in a high ${ }^{14} \mathrm{~N} /{ }^{16} \mathrm{O}$ ratio (in the $60 M_{\odot}$ star, ${ }^{14} \mathrm{~N}$ is depleted from He-burning in the innermost layers, resulting in a slightly lower ${ }^{14} \mathrm{~N} /{ }^{16} \mathrm{O}$ ratio than in the $20 M_{\odot}$ case). Subsequently, in the $20 M_{\odot}$ star the shock moves rapidly through the small remaining stellar envelope $\left(3.4 M_{\odot}\right)$ and starts propagating into the ISM, thus decreasing its mass-integrated ${ }^{14} \mathrm{~N} /{ }^{16} \mathrm{O}$ ratio rapidly. In the $60 M_{\odot}$ star, the shock runs through $\sim 20 M_{\odot}$ of processed material, with a high value of ${ }^{14} \mathrm{~N} /{ }^{16} \mathrm{O}$, before reaching the ISM; the corresponding ${ }^{14} \mathrm{~N} /{ }^{16} \mathrm{O}$ ratio decreases more slowly than in the $20 M_{\odot}$ case.

The evolution of ${ }^{22} \mathrm{Ne} /{ }^{20} \mathrm{Ne}$ is quite different in the two models. In the $20 M_{\odot}$ star, no He-burning products are encountered by the shock wave and the ${ }^{22} \mathrm{Ne} /{ }^{20} \mathrm{Ne}$ ratio always has its initial (solar) value. A high ${ }^{22} \mathrm{Ne} /{ }^{20} \mathrm{Ne}$ ratio is initially encountered in the processed layers of the $60 M_{\odot}$ star, which is progressively diluted as the shock moves outwards.

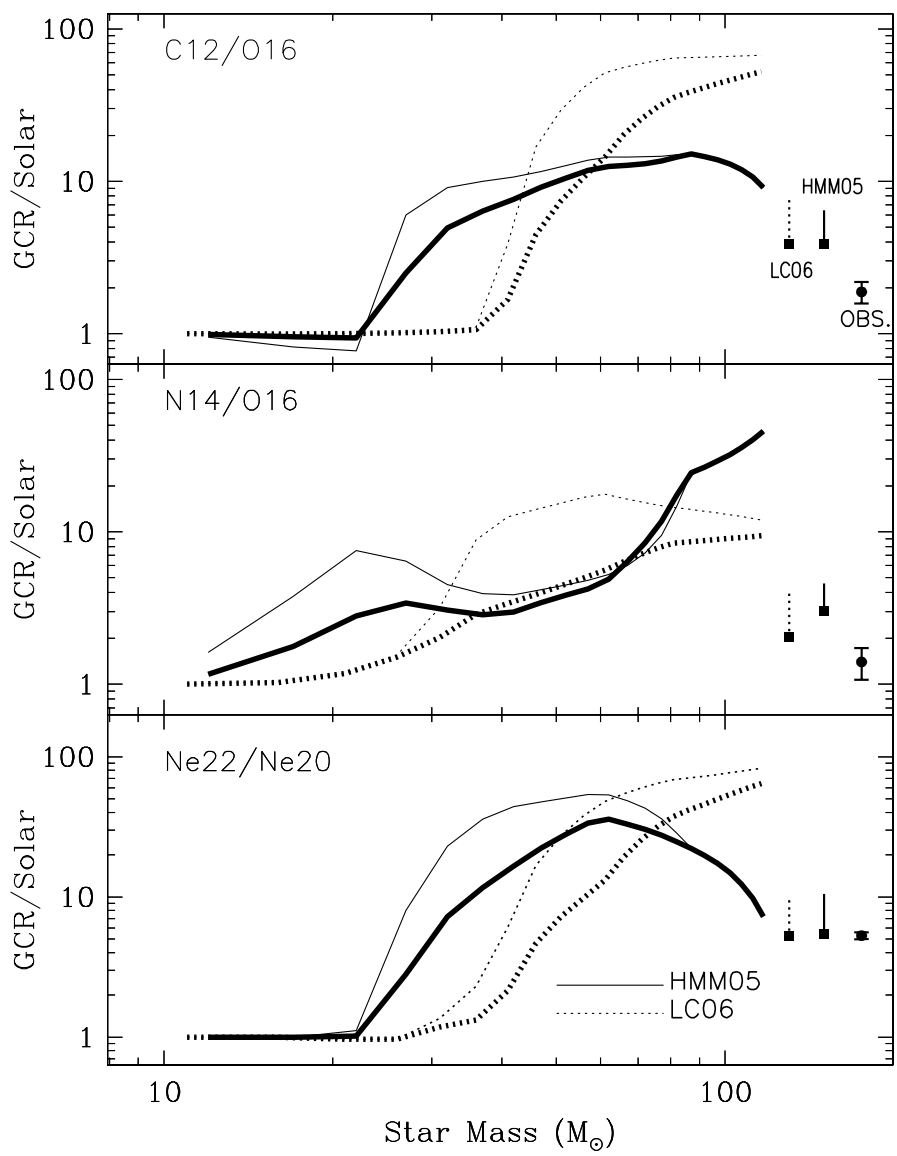

Fig. 6. Abundance ratios of various nuclear species in GCR source normalized to the corresponding solar ones as a function of the initial stellar mass. In all panels, solid curves correspond to models of HMM05 and dotted curves to models of LC06. Upper, thin curves are for GCR accelerated at the beginning of the ST phase and lower, thick curves for the time-average at the end of GCR acceleration. An average over a Salpeter IMF (and accounting for the swept-up mass in each case) produces the vertical segments to the right, their top point corresponding to the beginning and the bottom one to the end of the GCR acceleration phase, respectively (also indicated by filled squares). These results are compared to GCR source abundance ratios as derived by ACE data (points at the extreme right with error bars) in Binns et al. (2005). The most significant, unaffected by FIP, volatility etc., is the one of ${ }^{22} \mathrm{Ne} /{ }^{20} \mathrm{Ne}$. The end of the GCR acceleration phase is assumed to correspond to shock velocities $v_{\min }$ such that the time and IMF averaged theoretical ratio (squares) of ${ }^{22} \mathrm{Ne} /{ }^{20} \mathrm{Ne}$ matches the observed one (see text). For the set-up adopted here we find $v_{\min }=1900 \mathrm{~km} \mathrm{~s}^{-1}$ for HMM models and $v_{\min }=2400 \mathrm{~km} \mathrm{~s}^{-1}$ for LC06 models.

\subsection{Composition of accelerated particles}

The composition curves of the bottom panel of Fig. 5 give the time (or mass) integrated composition encountered by the shock wave and, in consequence, the composition of the particles that have been accelerated up to that time. We assume here that particles are accelerated to GCR energies with the same efficiency for shock velocities higher than some critical value $v_{\min }$, which is the same for all stellar masses. We determine $v_{\min }$ empirically by requiring that, when averaged over a stellar Initial mass function (IMF) $\Phi\left(M_{*}\right)$, the ratio

$\frac{M_{22}}{M_{20}}=\frac{\int_{10 M_{\odot}}^{120 M_{\odot}} \mathrm{d} M_{*} m_{22}\left(M_{*}\right) \Phi\left(M_{*}\right)}{\int_{10 M_{\odot}}^{120 M_{\odot}} \mathrm{d} M_{*} m_{20}\left(M_{*}\right) \Phi\left(M_{*}\right)}=R_{\mathrm{Obs}} \frac{X_{22, \odot}}{X_{20, \odot}}$ 
where $R_{\text {Obs }}=5.3 \pm 0.3$ is the observationally determined source GCR ratio of ${ }^{22} \mathrm{Ne} /{ }^{20} \mathrm{Ne}$ in solar units and $m_{22}\left(M_{*}\right)$ and $m_{20}\left(M_{*}\right)$ are calculated from Eq. (12) for stars of mass $M_{*}$ and for sweptup masses $M_{\mathrm{S}}\left(v>v_{\min }\right)$.

We adopt here a Salpeter IMF $\Phi\left(M_{*}\right) \propto M_{*}^{-X}$ with $X=2.35$. The results of the procedure appear in Fig. 6 for a few selected abundance ratios and for the models of both HMM05 (solid curves) and LC06 (dotted curves). In all panels, the upper (thin) curves correspond to the composition accelerated at the beginning of the ST phase (maximal possible deviations from solar composition). Stars with mass $<22 M_{\odot}$ (for HMM05) and $32 M_{\odot}$ (for LC06) display no He-burning products in their accelerated particles. $\mathrm{N}$ is overabundant in lower stellar masses, because of the 1st dredge-up.

The lower (thick) curves in all panels of Fig. 6 correspond to a composition accelerated up to the end of the acceleration period which is assumed to occur for a shock velocity $v_{\min }$. The corresponding IMF-averaged quantities (between 10 and $\left.120 M_{\odot}\right)$ are displayed to the right of the curves: their uppermost point corresponds to the beginning of the ST phase and the lower one (also indicated with a filled square) to the end of the acceleration period, i.e. to $v_{\min }$. The value of $v_{\min }$ is found to be $\sim 1900 \mathrm{~km} \mathrm{~s}^{-1}$ for the HMM05 models with rotation and $\sim 20 \%$ higher $\left(2400 \mathrm{~km} \mathrm{~s}^{-1}\right)$ for the LC06 models without rotation. The reason for that difference is, of course that rotating models have thicker processed layers, requiring more dilution with circumstellar material.

The top and middle panels of Fig. 6 display the corresponding ratios for ${ }^{12} \mathrm{C} /{ }^{16} \mathrm{O}$ and ${ }^{14} \mathrm{~N} /{ }^{16} \mathrm{O}$, respectively. In both cases, the IMF averaged ratios are higher than the observed ones in GCR (which are, in turn, higher than solar), by factors of $1.5-2$. Unlike ${ }^{22} \mathrm{Ne} /{ }^{20} \mathrm{Ne}$, these are ratios of different elements with different atomic properties. The GCR source abundances are known to be affected by atomic effects, e.g. First ionization potential (FIP), or, perhaps more plausibly, volatility and mass/charge ratio (see extensive discussion in Meyer et al. 1997). The analysis of these effects is beyond the scope of this study. We simply notice here that observations indicate that refractory elements are relatively more abundant in GCR sources than volatiles. Meyer et al. (1997) attribute that to the fact that refractories are locked up in grains, which are sputtered by the shock wave and the released ions are easily picked up and accelerated. Because ${ }^{16} \mathrm{O}$ is more refractory than both ${ }^{14} \mathrm{~N}$ and ${ }^{12} \mathrm{C}$, it is expected that its abundance in GCR source will be enhanced by that effect, and the corresponding ${ }^{12} \mathrm{C} /{ }^{16} \mathrm{O}$ and ${ }^{14} \mathrm{~N} /{ }^{16} \mathrm{O}$ ratios in GCR source will be lower than predicted from stellar nucleosynthesis alone. The fact that we obtain higher than observed ratios for ${ }^{12} \mathrm{C} /{ }^{16} \mathrm{O}$ and ${ }^{14} \mathrm{~N} /{ }^{16} \mathrm{O}$ in Fig. 6 is encouraging in that respect, because the aforementioned atomic effects would lower those ratios, hopefully to their observed values ${ }^{2}$.

\subsection{Efficiency of particle acceleration}

The material of Sects. 3.1 and 3.2 is summarized in Fig. 7 for the rotating models of HMM05. The forward shock, launched at mass coordinate $M_{\text {Exp }}$, sweeps up a mass $M_{S 1} \sim M_{\text {Exp }}$ and then starts accelerating particles, at mass coordinate $A_{1}=M_{\mathrm{Exp}}+M_{\mathrm{S} 1}$, up to point $A_{2}$ (where its velocity becomes $v_{\text {min }}$ ). For rotating

\footnotetext{
${ }^{2}$ One might think that a combined analysis of the ${ }^{12} \mathrm{C} /{ }^{16} \mathrm{O}$ and ${ }^{14} \mathrm{~N} /{ }^{16} \mathrm{O}$ ratios of Fig. 6 could help to constrain the atomic processes shaping the GCR source abundances. However, the abundances of ${ }^{12} \mathrm{C}$ and ${ }^{16} \mathrm{O}$ are affected by the still uncertain value of the $\left.{ }^{12} \mathrm{C}(\alpha, \gamma)\right)^{16} \mathrm{O}$ reaction rate and are unsuitable for such a study.
}

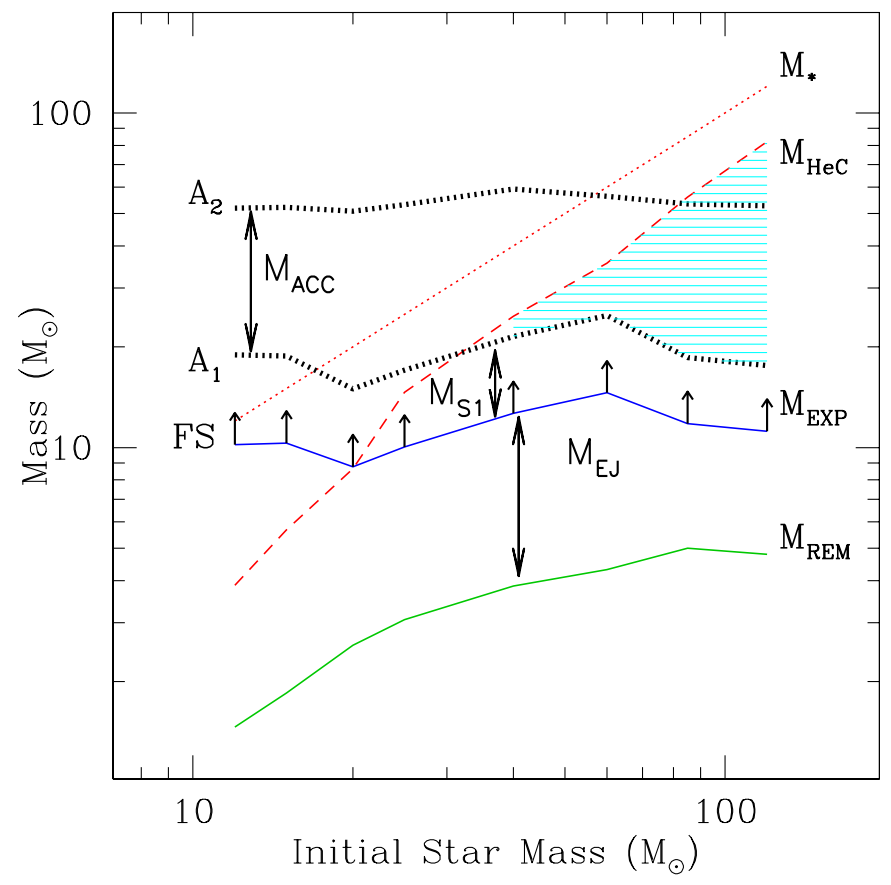

Fig. 7. Graphical presentation of the results discussed in Sect. 3.3. Particle acceleration starts at the beginning of the ST phase, located at mass coordinate $A_{1}=M_{\mathrm{Exp}}+M_{\mathrm{Ej}}$, i.e. when the forward shock (FS, $a r$ rows), launched at $M_{\mathrm{Exp}}$, has swept up a mass $M_{\mathrm{S} 1}=M_{\mathrm{Ej}}$. Acceleration stops at mass coordinate $A_{2}$, corresponding in the case dicussed here to a shock velocity of $1900 \mathrm{~km} \mathrm{~s}^{-1}$. The mass sampled by the FS between those two regions is $M_{\mathrm{Acc}}=A_{2}-A_{1}$. For rotating stars with mass $M>30 M_{\odot}$, an increasing part of $M_{\text {Acc }}$ includes nuclearly processed material (shaded aerea), while for rotating stars with $M<18 M_{\odot}, M_{\mathrm{ACcc}}$ contains only material of ISM (=solar) "composition".

stars with $M<15 M_{\odot}, A_{1}$ lies beyond the stellar surface and only ISM is accelerated. In stars with $15<M_{*} / M_{\odot}<25, A_{1}$ lies beyond the processed/mixed interior $M_{\mathrm{HeC}}$ and the forward shock first accelerates envelope and then ISM material. For stars above $35 M_{\odot}$, the shock first accelerates processed material (hatched aerea), then the $-{ }^{22} \mathrm{Ne}$ normal - envelope and then ISM. Finally, for stars with $M>70 M_{\odot}$, particle acceleration ends when the shock is still within the massive stellar envelope.

The mass of circumstellar material from which particles are accelerated is $M_{\mathrm{ACC}}=A_{2}-A_{1}$ and lies in the range 30-40 $M_{\odot}$. The mass of particles that have been accelerated is given by

$m_{A P}=\sum N_{i} A_{i} m_{\mathrm{P}}$

where $N_{i}$ is the total number of nuclei of species $i, A_{i}$ the corresponding mass number and $m_{\mathrm{P}}$ the proton mass. The number $N_{i}$, or rather the product $N_{i} A_{i}$, can be determined by noticing that the total energy carried by those accelerated particles is a fraction $f$ of the kinetic energy $E_{0}$ of the supernova

$\sum N_{i} A_{i} \int_{0}^{\infty} E Q(E) \mathrm{d} E=f E_{0}$,

where $Q(E)$ is the spectrum of accelerated particles $\left(A_{i}\right.$ appears on the left side of Eq. (15) because energies are expressed in energy units per nucleon). The efficiency $f$ of conversion of $E_{0}$ to accelerated particles escaping the supernova is estimated to $f \sim 0.1$, while another $0.1-0.2$ goes to the acceleration of particles that are finally trapped in the SN (Ellison \& Bykov 2011). 
The particle spectrum is often described by a power law in momentum

$Q(E) \propto \frac{p^{-s}}{\beta} \exp \left(-E / E_{\mathrm{C}}\right)$

where $\beta=v / c$ is the velocity expressed as a fraction of the light velocity, $p$ is the particle momentum per nucleon, the factor $s$ is usually $2<s<3$ (for strong shocks) and $E_{\mathrm{C}}$ is a cut-off energy, here taken to be $3 \mathrm{TeV}$ (the results are insensitive to much higher values).

Assuming that the spectrum of accelerated particles escaping the $\mathrm{SN}$ is given by Eq. (16), one finds the efficiency with which particles are accelerated from the shocked circumstellar medium, through Eqs. (14) to (16):

$W=\frac{m_{\mathrm{AP}}}{M_{\mathrm{ACC}}}$,

and for rotating stars it is found to be in the range $3-6 \times 10^{-6}$ i.e. a few particles out of a million encountered ones are accelerated by the forward shock to GCR energies. For non-rotating stars, the energetics is the same, but the swept-up mass is smaller (by a factor of two, on average, to obtain the observed ${ }^{22} \mathrm{Ne} /{ }^{20} \mathrm{Ne}$ ratio) and the corresponding efficiency is $W \sim 10^{-5}$. These estimates constitute only a gross average, since the efficiency of particle acceleration may depend on several factors, not considered here, such as the density of the circumstellar medium or the shock velocity - through a smoothly varying function $f\left(v_{\mathrm{S}}\right)$ instead of the Heavyside function $f\left(>v_{\min }\right)=1$ and $f\left(<v_{\min }\right)=0$ considered here - or the shock radius at the time of acceleration, since particles may subsequently suffer adiabatic cooling before escaping (e.g. Drury 2011; Bykov \& Ellison 2011). Notice that some of those effects may have opposite time dependencies. For instance, particles accelerated earlier on (at higher shock velocities and presumably with higher efficiencies) are expected to suffer more from adiabatic cooling (because they are produced at smaller radii). These effects require a much more thorough investigation. As a first step in that direction, we also tested the case where the efficiency of shock acceleration varies with shock velocity as $f \propto v^{2}$ (Drury 2011). In that case, material with high ${ }^{22} \mathrm{Ne} /{ }^{20} \mathrm{Ne}$ is efficiently accelerated in the inner layers of the most massive stars. To obtain again the observed GCR source ${ }^{22} \mathrm{Ne} /{ }^{20} \mathrm{Ne}$ ratio, one has to dilute the mixture by allowing acceleration for shock velocities lower then the reference values found above: we thus find values of $v_{\min }=1600 \mathrm{~km} \mathrm{~s}^{-1}$ for the HMM05 yields and $2150 \mathrm{~km} \mathrm{~s}^{-1}$ for the LC06 yields; the corresponding overall acceleration efficiencies increase then by $\sim 40 \%$ from the reference values given above, ranging from $4-7 \times 10^{-6}$ for HMM05 yields to $1-1.4 \times 10^{-5}$ for LC06 yields. The reason for obtaining such a small difference (only a few hundreds of $\mathrm{km} \mathrm{s}^{-1}$ ) between the non-realistic reference case and the perhaps more realistic - case of velocity dependent efficiency, is the adopted unified treatment, which considers acceleration in both low-mass and high-mass supernova: the former accelerate almost pure ISM with low ${ }^{22} \mathrm{Ne} /{ }^{20} \mathrm{Ne}$ and the latter almost pure wind material with high ${ }^{22} \mathrm{Ne} /{ }^{20} \mathrm{Ne}$. Allowing for lower values of $v_{\text {min }}$ involves a considerably larger amount of ISM processed by low-mass supernovae, since the lower acceleration efficiency $\left(f \propto v^{2}\right)$ is more than compensated for by the $n_{\mathrm{ISM}} 4 \pi r^{2} \mathrm{~d} r$ factor.

As approximate as they may be, the results obtained here through the constraint of the observed GCR source ${ }^{22} \mathrm{Ne} /{ }^{20} \mathrm{Ne}$ ratio, clearly indicate that acceleration has to occur for shock velocities higher than $\sim 1500 \mathrm{~km} \mathrm{~s}^{-1}$, and they may help to improve our understanding of particle acceleration in SN remnants.

\section{GCR cannot be accelerated (mainly) in superbubbles}

The idea that CGR are accelerated mainly in superbubbles has been suggested by Kafatos et al. (1981) and reassessed by Higdon et al. (1998). Massive stars are mainly formed in OB associations and the winds of the most massive of them initiate the formation of superbubbles, while the subsequent SN explosions power the expansion of those superbubbles for a few $10^{7}$ years. Higdon et al. (1998) argued that the environment of these superbubbles, enriched with the ejecta of stellar winds and core collapse SN explosions, provides a composition that compares favourably to the inferred GCR source composition.

One potential problem with that idea is that ${ }^{59} \mathrm{Ni}$, a wellknown product of SN nucleosynthesis, is absent from GCR arriving on Earth (Wiedenbeck et al. 1999). Since ${ }^{59} \mathrm{Ni}$ is unstable to electron capure, with a lifetime of $\sim 10^{5} \mathrm{y}$, Higdon et al. (1998) argued that $\mathrm{SN}$ explosions occur within the superbubble with a sufficiently low frequency (less than one SN every $3 \times 10^{5} \mathrm{y}$ ), to allow for the decay of ${ }^{59} \mathrm{Ni}$ between two SN explosions. Prantzos (2005) pointed out that energetic stellar winds can also accelerate particles and they are not intermittent (like SN) but occur continuously in superbubbles; in that case ${ }^{59} \mathrm{Ni}$ is continuously accelerated to GCR energies and, being unable to capture an electron, it becomes effectively stable and it should be detectable in GCR. Binns et al. (2008) counter-argued that the period of energetic WR winds represents a small (albeit not negligible) fraction in the early lifetime of an OB association and therefore only little (and presumably undetectable) amounts of ${ }^{59} \mathrm{Ni}$ would be accelerated, thus saving the "superbubble paradigm".

In the meantime, Higdon \& Lingenfelter (2003) evaluated the isotopic composition of ${ }^{22} \mathrm{Ne} /{ }^{20} \mathrm{Ne}-$ the critical abundance anomaly in GCR - expected in a superbubble, based on (a very heterogeneous set of) then available yields of WR stars and SN. They found that the GCR ${ }^{22} \mathrm{Ne} /{ }^{20} \mathrm{Ne}$ ratio "can be easily understood as the result of GCR accelerated primarily out of superbubbles with a mean metallicity $Z_{\mathrm{SB}}=2.7 \pm 0.4 Z_{\odot}$ " and that this result "provides strong, additional evidence for a superbubble origin of GCR". In a subsequent paper, Lingenfelter \& Higdon (2007) quantitatively explored that senario for other GCR abundances, finding again that results compare favourably to observations.

Despite their apparent sophistication, the aforementioned arguments for a superbubble origin of GCR miss a simple point: massive stars are the principal source of both ${ }^{20} \mathrm{Ne}$ and ${ }^{22} \mathrm{Ne}$ in the Universe. As a result, the ${ }^{22} \mathrm{Ne} /{ }^{20} \mathrm{Ne}$ ratio of a generation of massive stars (integrated over an IMF and including WR winds and all kinds of SN ejecta) should be solar ${ }^{3}$. Because ${ }^{22} \mathrm{Ne}$ is overabundant with respect to ${ }^{20} \mathrm{Ne}$ only in the massive star winds (and not in the SN ejecta or the ISM), a popular exercise in the past 30 years or so consisted in evaluating the mixing ratio of WR ejecta with SN ejecta (or with average ISM), as to obtain the observed ${ }^{22} \mathrm{Ne} /{ }^{20} \mathrm{Ne}$ ratio in GCR (see Introduction and references therein).

However, it is expected that a properly weighted mixture of the ejecta of massive stars in a superbubble (i.e. including stellar winds and SN ejecta and folded with a stellar IMF) would produce a solar ${ }^{22} \mathrm{Ne} /{ }^{20} \mathrm{Ne}$ ratio. In this section, the same exercise is repeated with the yields of LC06 and HMM05 and by taking into

\footnotetext{
${ }^{3}$ Strictly speaking, this concerns massive stars of roughly solar metallicity, such as those that contributed to the composition of the solar system; however, metallicity has evolved very little in the past several billion years in the local volume of $\sim 1-2 \mathrm{kpc}$ radius, where most GCR originate.
} 


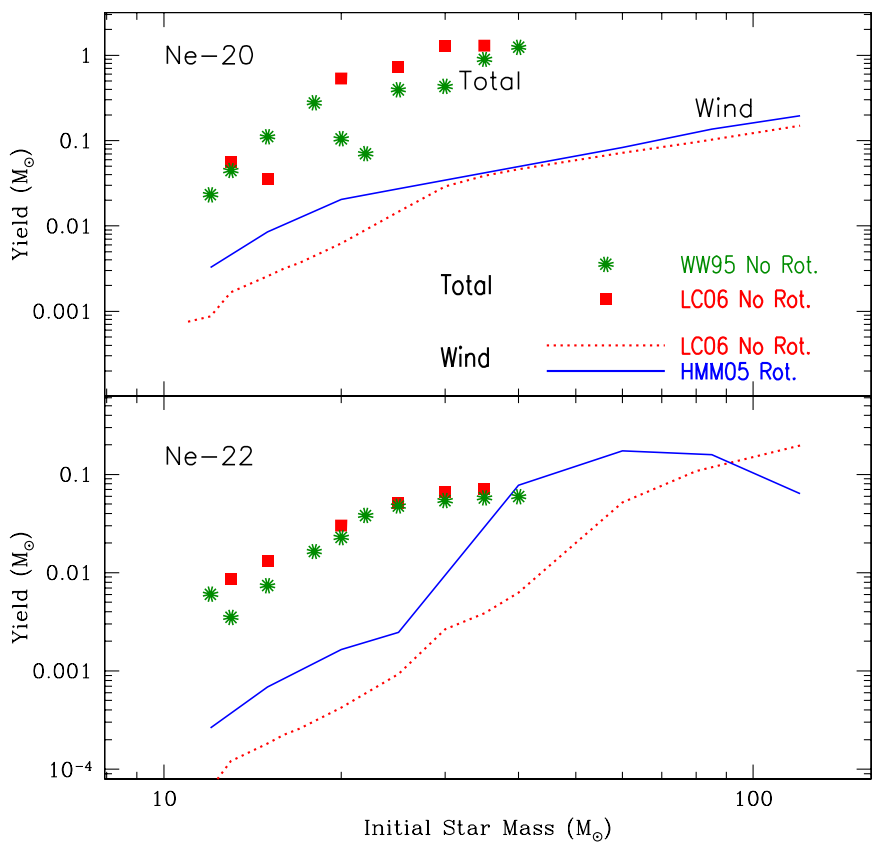

Fig. 8. Yields of ${ }^{20} \mathrm{Ne}$ (top) and ${ }^{22} \mathrm{Ne}$ (bottom) as a function of the initial stellar mass. Wind yields are from HMM05 (solid curves) and LC06 (dotted curves), respectively (same as in Fig. 3). Total yields are from LC06 (filled squares) and WW95 (asterisks).

account the corresponding stellar lifetimes. In Fig. 8 we present again the adopted ${ }^{20} \mathrm{Ne}$ and ${ }^{22} \mathrm{Ne}$ yields of LC06 and HMM05 for the stellar winds (solid and dotted curves, i.e. the same as in Fig. 3) and also the total yields (winds plus SN ejecta, points). The latter are from LC06 for stars with mass loss (but no rotation) and from Woosley \& Weaver (1995, WW95) for stars without mass loss; they agree well overall, except for ${ }^{20} \mathrm{Ne}$ in stars around $20 M_{\odot}$. For the SN ejecta, we consider only stars with $M<40 M_{\odot}$, i.e. we assume that more massive stars eject their ${ }^{20} \mathrm{Ne}$ and ${ }^{22} \mathrm{Ne}$ through their winds and then form black holes; in that case we maximize the ${ }^{22} \mathrm{Ne} /{ }^{20} \mathrm{Ne}$ ratio expected from massive stars.

An inspection of Fig. 8 shows that the total (wind $+\mathrm{SN}){ }^{22} \mathrm{Ne}$ yields of $M<40 M_{\odot}$ stars are comparable to the wind ${ }^{22} \mathrm{Ne}$ yields of the most massive $\left(\sim 100 M_{\odot}\right)$ stars; however, the corresponding total ${ }^{20} \mathrm{Ne}$ yields of $M<40 M_{\odot}$ stars are at least 10 times higher than the wind ${ }^{20} \mathrm{Ne}$ yields of the most massive $\left(\sim 100 M_{\odot}\right)$ stars. Thus, while the production of ${ }^{22} \mathrm{Ne}$ receives a sizeable contribution from the most massive stellar winds (at least for the rotating stars), the production of ${ }^{20} \mathrm{Ne}$ is totally dominated by the $\mathrm{SN}$ ejecta of $M<40 M_{\odot}$ stars. In a coeval stellar population, like the one expected in an $\mathrm{OB}$ association or a superbubble, the ${ }^{22} \mathrm{Ne} /{ }^{20} \mathrm{Ne}$ ratio will evolve then from higher than solar to $\sim$ solar values, as the $M<40 M_{\odot}$ stars eject their core products a few Myr after their more massive counterparts.

We quantitative illustrate this effect in Fig. 9, where we present the evolution of a stellar population born at time $t=0$ with a Salpeter IMF and total mass of $1 M_{\odot}$ (results can be directly scaled to masses of $\mathrm{OB}$ associations, while abundances and abundance ratios remain the same). The top right panel displays the rate of $\mathrm{SN}$ explosions; for a total stellar mass of $10^{4} M_{\odot}$, resulting in a $\sim 100 \mathrm{OB}$ stars, there are about $3 \mathrm{SN} / \mathrm{Myr}$, i.e. $\sim 1 \mathrm{SN}$ every $300000 \mathrm{yr}$, as evaluated in Higdon et al. (1998) to avoid the problem of ${ }^{59} \mathrm{Ni}$ acceleration. The top left panel displays the ejection rates of ${ }^{20} \mathrm{Ne}$ and ${ }^{22} \mathrm{Ne}$ (in $M_{\odot} /$ Myr per $M_{\odot}$ of stars formed): ${ }^{22} \mathrm{Ne}$ from stellar winds dominates only for a

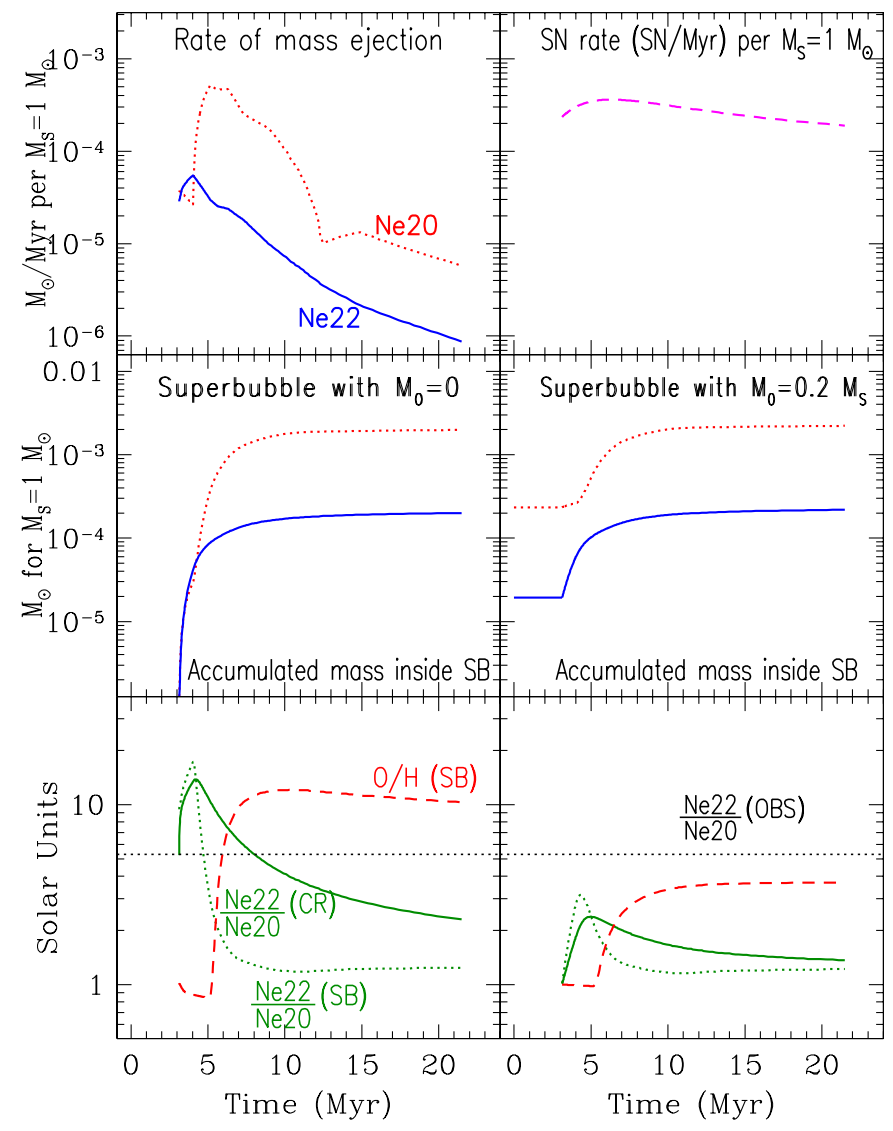

Fig. 9. Evolution of composition in a superbubble. Top left: ejection rate of ${ }^{20} \mathrm{Ne}($ dotted $)$ and ${ }^{22} \mathrm{Ne}($ solid $)$ in $M_{\odot} / \mathrm{Myr}$ per $1 M_{\odot}$ of stars formed (assuming a Salpeter IMF between 0.1 and $120 M_{\odot}$ ). Top right: supernova rate per Myr. Middle: total mass of ${ }^{20} \mathrm{Ne}$ (dotted) and ${ }^{22} \mathrm{Ne}$ (solid) accumulated in the superbubble for no initial gas $\left(M_{0}=0\right.$, left $)$ and for an initial mass of gas equal to $1 / 5$ of the formed stars $\left(M_{0}=0.20\right.$, right); in the former case ${ }^{22} \mathrm{Ne}$ dominates by mass early on, whereas in the latter the mass of ${ }^{20} \mathrm{Ne}$ dominates from the very beginning. Bottom: corresponding evolution of the $\mathrm{O} / \mathrm{H}$ (dashed) and of the $\left({ }^{22} \mathrm{Ne} /{ }^{20} \mathrm{Ne}\right)_{\mathrm{SB}}$ ratio (dotted) in the $\mathrm{SB}$ gas; the solid curve represents the $\left({ }^{22} \mathrm{Ne} /{ }^{20} \mathrm{Ne}\right)_{\mathrm{CR}}$ ratio of particles accelerated up to that time and has to be compared to the observed GCR source ratio (dotted horizontal line).

couple of Myr but $\sim 5 \mathrm{Myr}$ after the stellar formation, ${ }^{20} \mathrm{Ne}$ from $M<40 M_{\odot}$ explosions dominates. Notice that, to maximise the ${ }^{22} \mathrm{Ne} /{ }^{20} \mathrm{Ne}$ ratio, we adopted only the yields of HMM05 for these calculations (i.e. corresponding to the solid curves in Fig. 8 with wind yields only above $M=40 M_{\odot}$ ).

The instantaneous $\left({ }^{22} \mathrm{Ne} /{ }^{20} \mathrm{Ne}\right)_{S B}$ ratio in the superbubble at time $t$ is the ratio of the amounts of ${ }^{20} \mathrm{Ne}$ and ${ }^{22} \mathrm{Ne}$ cumulated up to that time, which are provided in the middle panels of Fig. 9. To evaluate those quantities, one needs to make an assumption about the amount of pre-existing gas still left inside the superbubble. In the left middle panel of Fig. 9 it is assumed that no gas is left after star formation: gas is exclusively supplied by the wind and explosive stellar ejecta. In that case, ${ }^{22} \mathrm{Ne}$ dominates early on, but ${ }^{20} \mathrm{Ne}$ takes over after a couple of Myr. In the right middle panel it is assumed that an amount of gas with solar composition and equal to $20 \%$ of the mass of formed stars remained in the superbubble; the stellar ejecta are diluted into it and the superbubble composition is now dominated always by ${ }^{20} \mathrm{Ne}$.

The bottom panels of Fig. 9 display the corresponding $\left({ }^{22} \mathrm{Ne} /{ }^{20} \mathrm{Ne}\right)_{\mathrm{SB}}$ ratios in the superbubble. For zero initial gas (left), the instantaneous $\left({ }^{22} \mathrm{Ne} /{ }^{20} \mathrm{Ne}\right)_{\mathrm{SB}}$ ratio remains high - from 
18 to 5 times solar - for a couple of Myr, but soon after stars of $M<40 M_{\odot}$ start dying, its value decreases rapidly to solar (as expected from the nucleosynthesis argument presented in the beginning of this section). The $\left({ }^{22} \mathrm{Ne} /{ }^{20} \mathrm{Ne}\right)_{\mathrm{CR}}(<t)$ ratio of particles accelerated to CR energies up to time $t$ is the time integral of the instantaneous $\left({ }^{22} \mathrm{Ne} /{ }^{20} \mathrm{Ne}\right)_{\mathrm{SB}}$ ratio in the superbubble

$$
\left(\frac{{ }^{22} \mathrm{Ne}}{{ }^{20} \mathrm{Ne}}\right)_{\mathrm{CR}}(<t)=\frac{1}{t} \int_{0}^{t}\left(\frac{{ }^{22} \mathrm{Ne}}{{ }^{20} \mathrm{Ne}}\right)_{\mathrm{SB}} \mathrm{d} t^{\prime}
$$

where it is assumed that the efficiency of particle acceleration does not vary with time. $\left({ }^{22} \mathrm{Ne} /{ }^{20} \mathrm{Ne}\right)_{\mathrm{CR}}(<t)$ represents the mean CR composition accelerated by a superbubble which "operates" up to time $t$.

In the bottom left panel it is seen that the $\left({ }^{22} \mathrm{Ne} /{ }^{20} \mathrm{Ne}\right)_{\mathrm{CR}}(<t)$ ratio (in solar units) remains above the observed $R_{\mathrm{Obs}}=5.3 \pm 0.3$ for about $7 \mathrm{Myr}$, while it declines steadily after that, tending to an asymptotic value of $\sim 2$, considerably smaller than observed. The corresponding metallicity in the superbubble, expressed by the $\mathrm{O} / \mathrm{H}$ ratio (dashed curve), is quite high - about 10 times solar - since it represents pure core collapse SN ejecta; such high metallicity values have never been reported for any astrophysical environment (except SN remnants).

The bottom right panel of Fig. 9 displays the corresponding quantities in the more realistic case of a superbubble endowed with some gas left over from the star formation. In that case, although metallicity is still high (about 3 times solar after $7 \mathrm{Myr}$ ), the ${ }^{22} \mathrm{Ne} /{ }^{20} \mathrm{Ne}$ ratios never get above 3 times solar, either in the superbubble or in the accelerated particles; in fact they are close to solar for the largest part of the superbubble lifetime.

The results displayed in Fig. 9 suggest that the observed $R_{\text {Obs }}=5.3 \pm 0.3$ ratio in GCR cannot be obtained from material accelerated in a superbubble: even under the most favorable possible conditions, such as those adopted here (yields from rotating massive stars, only wind yields considered above $M=40 M_{\odot}$, no gas left over from star formation), the resulting ${ }^{22} \mathrm{Ne} /{ }^{20} \mathrm{Ne}$ ratio remains at high values only in the early evolution of the superbubble, during a small fraction of its lifetime. Interestingly, Binns et al. (2008) suggested that no substantial particle acceleration must occur during that early period to avoid the problem of ${ }^{59} \mathrm{Ni}$ acceleration from the WR winds; thus, saving the "superbubble paradigm" for the origin of cosmic rays requires that acceleration occurs only in the late superbubble evolution, when the WR winds have essentially stopped and only SN inject intermittently their kinetic energy in the superbubble. However, we have shown here that during this period, the $\left({ }^{22} \mathrm{Ne} /{ }^{20} \mathrm{Ne}\right)_{\mathrm{CR}}$ ratio is considerably lower than observed. Indeed, under realistic conditions (some initial gas left, explosive yields also above $40 M_{\odot}$ considered, at least for some stars), the ${ }^{22} \mathrm{Ne} /{ }^{20} \mathrm{Ne}$ ratio in a superbubble would never reach values as high as observed, contrary to claims made in the literature in the past decade. It must be stressed that this conclusion does not depend on detailed adopted yields, but on a simple argument, namely that the IMF averaged ${ }^{22} \mathrm{Ne} /{ }^{20} \mathrm{Ne}$ ratio in a superbubble has to be close to solar during the longest period of the superbubble lifetime.

\section{Summary}

We explore some implications of the idea that cosmic rays are accelerated by the forward shock in supernova remnants during their ST phase. We focus on the chemical composition resulting from such an acceleration and, in particular, on the ${ }^{22} \mathrm{Ne} /{ }^{20} \mathrm{Ne}$ ratio, which is the most characteristic feature of the observed GCR source composition and is unaffected by atomic effects.
For that purpose, we adopt recent models of the nucleosynthesis and evolution of massive stars with mass loss: those of LC06 with no rotation and those of HMM05 with rotation.

In Sect. 2 we present a detailed summary of the properties of those models and, in particular, of the chemical composition of their winds, insisting that rotating models release more ${ }^{22} \mathrm{Ne}$ in their winds than non-rotating ones. We also present the adopted model for the evolution of a SN remnant within a stellar wind, based on the ideas of Ptuskin \& Zirakashvili (2005) and the equations summarized in Caprioli (2011).

In the framework of the simple model adopted here, we follow the time-dependent composition of GCR, accelerated by the forward shock as it runs through either the ISM (for stars with $M<25-35 M_{\odot}$, depending on rotation) or through the stellar wind (for more massive stars). Indeed, during the longest part of the ST phase, the shock runs through ISM and encounters a solar composition. In order to reproduce the observed high value of $\left({ }^{22} \mathrm{Ne} /{ }^{20} \mathrm{Ne}\right)_{\mathrm{CR}}\left(R_{\mathrm{Obs}}=5.3 \pm 0.3\right.$ in solar units $)$ after accounting for the stellar IMF, we have then to assume that acceleration is efficient only during a short early period in the ST phase. We chose to use the shock velocity as a criterion for efficient acceleration and, based on the aforementioned GCR composition argument, we find that shock velocities larger than $\sim 1900 \mathrm{~km} \mathrm{~s}^{-1}$ (for the rotating stellar models) or $2400 \mathrm{~km} \mathrm{~s}^{-1}$ (for the non-rotating ones) are required. This result is obtained by assuming a step function for the efficiency $f$ of particle acceleration $(f=0$ before the ST phase and after $v_{\text {min }}$, and $f=1$ between the two). For the - perhaps more realistic - assumption of a velocitydependent efficiency $f \propto v^{2}$, we find slightly lower values for $v_{\text {min }}\left(1600 \mathrm{~km} \mathrm{~s}^{-1}\right.$ for HMM05 yields and $2150 \mathrm{~km} \mathrm{~s}^{-1}$ for LC06 yields, respectively). In the framework of the adopted models, this corresponds to a circumstellar mass of several tens of $M_{\odot}$ encountered by the forward shock. Assuming, furthermore, that $10 \%$ of the SN kinetic energy is used in acceleration of escaping cosmic rays with standard energy spectra allowed us to evaluate the efficiency of that acceleration: we find that a few particles out of a million encountered by the forward shock are accelerated to CR energies. We also notice that this scheme of GCR acceleration does not suffer from problems related to the absence of unstable ${ }^{59} \mathrm{Ni}$ in observed GCR composition: this heavy nucleus is well inside the SN ejecta and is not reached by the forward shock, which accelerates only wind material and ISM.

The aforementioned scenario assumes that even the most massive stars, up to $120 M_{\odot}$, develop strong forward shocks and accelerate the particles of their WR winds. For non-rotating stars, this is probably an extreme assumption, because it has been argued (Heger et al. 2003) that non-rotating massive stars of about solar metallicity collapse into black holes, if their mass is in the 30-60 $M_{\odot}$ range (see their Fig. 1). Notice that stars in the $60-120 M_{\odot}$ range (the most important ${ }^{22} \mathrm{Ne}$ producers) end as black holes in their scheme. However, for slightly higher metallicities - such as those encountered in the inner Galactic disk - these authors find that only neutron stars are formed, because higher stellar mass losses result in a less massive star at explosion. It should be noticed that the details of massive star explosions remain poorly understood at present (see e.g. Hanke et al. 2011) and so is the fate of a massive star above $30 M_{\odot}$ (see Fryer et al. 2011, for a recent - but certainly not definitive - assessment). The fate of the rotating mass-losing stars considered here is even less well known.

In view of the aformentioned uncertainties, we feel that the scenario proposed here can be considered as valid at present, although future refinements in our understanding of massive star explosions may change it quantitatively (and even qualitatively, 
if it turns out that most masive stars above, say, $50 M_{\odot}$, end up as black holes).

Finally, we explore the idea that CR are accelerated in superbubbles, in which case their composition results from the ejecta of both stellar winds and SN explosions. We first notice that simple nucleosynthesis arguments suggest that the resulting composition, averaged over the stellar IMF, should be very close to solar. We demonstrate this quantitatively, with a simple model for the evolving composition of a superbubble, enriched first by the $\left({ }^{22} \mathrm{Ne}\right.$ rich) winds of the most massive stars, then by the $\left({ }^{20} \mathrm{Ne}\right.$ rich) SN ejecta of less massive stars. We find that, after a few Myr the superbubble $\left({ }^{22} \mathrm{Ne} /{ }^{20} \mathrm{Ne}\right)_{\mathrm{SB}}$ ratio tends to solar, and so does the average $\left({ }^{22} \mathrm{Ne} /{ }^{20} \mathrm{Ne}\right)_{\mathrm{CR}}$ ratio in accelerated particles. We conclude that superbubbles cannot provide the observed high $R_{\text {Obs }}$ value of GCR sources and, therefore, are not the main site of GCR acceleration. In contrast, SN remnants - including those expanding in the pre-explosion environment of a stellar wind appear to be suitable sites of GCR acceleration.

Acknowledgements. I am grateful to G. Meynet and M. Limongi for providing tables with yields and other properties of their stellar models and to the referee, L. Drury, for constructive remarks.

\section{References}

Anders, E., \& Grevesse, N. 1989, GeCoA, 53, 197

Asplund, M., Grevesse, N., Sauval, A. J., \& Scott, P. 2009, ARA\&A, 47, 481

Berezhko, E. G., \& Völk, H. J. 2006, A\&A, 451, 981

Berezhko, E. G., Pühlhofer, G., \& Völk, H. J. 2009, A\&A, 505, 641

Bibring, J.-P., \& Cesarsky, C. J. 1981, ICRC, 2, 289

Biermann, P. L., Langer, N., Seo, E.-S., \& Stanev, T. 2001, A\&A, 369, 269

Binns, W. R., Wiedenbeck, M. E., Arnould, M., et al. 2005, ApJ, 634, 351

Binns, W. R., Wiedenbeck, M. E., Arnould, M., et al. 2007, SSRv, 130, 439

Binns, W. R., Wiedenbeck, M. E., Arnould, M., et al. 2008, NewAR, 52, 427

Caprioli, D. 2011, J. Cosmol. Astropart. Phys., 05, 026

Caprioli, D., Amato, E., \& Blasi, P. 2010, APh, 33, 160

Caprioli, D., Blasi, P., \& Amato, E. 2011, Astropart. Phys., 34, 447

Casse, M., \& Goret, P. 1978, ApJ, 221, 703

Casse, M., \& Paul, J. A. 1982, ApJ, 258, 860

Drury, L. O'C. 2011, MNRAS, 415, 1807
Ellison, D. C., Drury, L. O'C., \& Meyer, J.-P. 1997, ApJ, 487, 197

Ellison, D. C., \& Bykov, A. M. 2011, ApJ, 731, 87

Fryer, C., Belczynski, K., Wiktorowicz, G., et al. 2011, ApJ, submitted [arXiv: 1110.1726$]$

Garcia-Munoz, M., Simpson, J. A., \& Wefel, J. P. 1979, ApJ, 232, L95

Garcia-Segura, G., Langer, N., \& Mac Low, M.-M. 1996a, A\&A, 316, 133

Garcia-Segura, G., Mac Low, M.-M., \& Langer, N. 1996b, A\&A, 305, 229

Hanke, F., Marek, A., Mueller, B., \& Janka, H.-T. 2011, ApJ, submitted [arXiv: 1108.4355]

Heger, A., Fryer, C. L., Woosley, S. E., Langer, N., \& Hartmann, D. H. 2003, ApJ, 591, 288

Higdon, J. C., \& Lingenfelter, R. E. 2003, ApJ, 590, 822

Hirschi, R., Meynet, G., \& Maeder, A. 2005, A\&A, 433, 1013

Kafatos, M., Bruhweiler, F., \& Sofia, S. 1981, 17th International Cosmic Ray Conference, 2, 222

Lingenfelter, R. E., \& Higdon, J. C. 2007, ApJ, 660, 330

Limongi, M., \& Chieffi, A. 2006, ApJ, 647, 483

Limongi, M., \& Chieffi, A. 2008, EAS, 32, 233

Lodders, K. 2003, ApJ, 591, 1220

Maeder, A. 1983, A\&A, 120, 130

Maeder, A., \& Meynet, G. 1993, A\&A, 278, 406

Maeder, A., \& Meynet, G. 2000, ARA\&A, 38, 143

Meyer, J.-P. 1985, ApJS, 57, 173

Meyer, J.-P., Drury, L. O., \& Ellison, D. C. 1997, ApJ, 487, 182

Ostriker, J. P., \& McKee, C. F. 1988, RvMP, 60, 1

Parizot, E., Marcowith, A., van der Swaluw, E., Bykov, A. M., \& Tatischeff, V. 2004, A\&A, 424, 747

Prantzos, N. 1984, AdSpR, 4, 109

Prantzos, N. 2005, in Chemical Abundances and Mixing in Stars in the Milky Way and its Satellites, ESO Astrophysics Symposia (Springer-Verlag), 351 [arXiv: astro-ph/0411569]

Prantzos, N. 2010, IAUS, 268, 473

Prantzos, N., Arnould, M., \& Arcoragi, J.-P. 1987, ApJ, 315, 209

Schaller, G., Schaerer, D., Meynet, G., \& Maeder, A. 1992, A\&AS, 96, 269

Ptuskin, V. S., \& Zirakashvili, V. N. 2005, A\&A, 429, 755

Ptuskin, V., Zirakashvili, V., \& Seo, E.-S. 2010, ApJ, 718, 31

Ramaty, R., Kozlovsky, B., Lingenfelter, R. E., \& Reeves, H. 1997, ApJ, 488, 730

Schure, K. M., Achterberg, A., Keppens, R., \& Vink, J. 2010, MNRAS, 406, 2633

Strong, A. W., Moskalenko, I. V., \& Ptuskin, V. S. 2007, ARNPS, 57, 285

Wiedenbeck, M. E., \& Greiner, D. E. 1981, PhRvL, 46, 682

Wiedenbeck, M. E., Binns, W. R., Christian, E. R., et al. 1999, ApJ, 523, L6

Wiedenbeck, M. E., Binns, W. R., Cummings, A. C., et al. 2007, SSRv, 130, 415

Woosley, S. E., \& Weaver, T. A. 1995, ApJS, 101, 181 